\title{
THE DYNAMIC COMPETITION HYPOTHESIS
}

\author{
by \\ Rehan Taj Malik \\ Bachelor in Science, University of Sindh, Pakistan, 1991 \\ Master of Science in Mathematics, QAU, Pakistan, 1995 \\ Master of Arts in Economics, University of Sindh, Pakistan, 1996 \\ Bachelor in Education, Preston University, Pakistan, 2015
}

\author{
A thesis presented to Ryerson University \\ in partial fulfillment of the \\ requirements for the degree of \\ Master of Science \\ in the program of \\ Applied Mathematics
}

Toronto, Ontario, Canada, 2018

(C) Rehan Malik, 2018 


\section{AUTHOR'S DECLARATION FOR ELECTRONIC SUBMISSION OF A THESIS}

I hereby declare that I am the sole author of this thesis. This is a true copy of the thesis, including any required final revisions, as accepted by my examiners.

I authorize Ryerson University to lend this thesis to other institutions or individuals for the purpose of scholarly research.

I further authorize Ryerson University to reproduce this thesis by photocopying or by other means, in total or in part, at the request of other institutions or individuals for the purpose of scholarly research.

I understand that my thesis may be made electronically available to the public. 


\title{
The Dynamic Competition Hypothesis
}

\author{
Master of Science, 2018 \\ Rehan Taj Malik \\ Applied Mathematics, Ryerson University
}

\begin{abstract}
We study competitive and negative interactions in real world social network in which nodes represent agents and edges appear over discrete time step. We consider directed social network of competing agents that evolve dynamically over time, where directed edges represent some kind of negative relationships between the agents in the social network.

We present a novel hypothesis to identify the alliances and leaders within the the dynamic competition networks. We verify our hypothesis by using historical voting data of the social game shows Survivor and Big Brother.
\end{abstract}




\section{Acknowledgements}

I would first like to thank my supervisor Dr. Anthony Bonato for giving me the opportunity to write a fulfilling thesis. Throughout my thesis-writing period, he provided encouragement, sound advice, excellent teaching, and lots of good ideas.

It is also a pleasure to thank Dr. Dejan Delić and Dr. Peter Danziger for serving as my committee members and for their valuable comments and suggestions.

Lastly and most importantly, I would like to thank my wife for her support and understanding throughout my graduate studies. I dedicate this thesis to my parents, Malik Taj Muhammad and Shamim Taj Malik. They raised me, supported me, taught me, and loved me. I could not be successful without their prayers. 


\section{TABLE OF CONTENTS}

Declaration $\quad$ ii

$\begin{array}{lll}\text { Abstract } & \text { iii }\end{array}$

Acknowledgements $\quad$ iv

List of Figures vii

List of Tables $\mathrm{x}$

Chapter 1. Introduction 1

1.1. Motivation 1

1.2. Introduction to Graph Theory 3

1.3. Summary of the Thesis 13

Chapter 2. The Dynamic Competition Hypothesis 14

2.1. Structural Balance Theory 14

2.2. Centrality metrics in networks 20

2.3. Dynamic Competition Networks 28

2.4. The Hypothesis 30

Chapter 3. Data and Methods 33

3.1. Survivor 33 
3.2. Big Brother 35

3.3. Social Network Analysis tools 36

$\begin{array}{ll}\text { 3.4. Borneo } & 37\end{array}$

3.5. China 40

3.6. Game Changers 42

3.7. Heroes vs Healers vs Hustlers (HHH) 44

3.8. Big Brother 47

3.9. Alliances 48

Chapter 4. Conclusion and Future Work 51

4.1. Conclusion 51

4.2. Future Work 52

$\begin{array}{ll}\text { Bibliography } & 54\end{array}$ 


\section{List of Figures}

1.1 $G$ has multiple edges and a loop and $H$ is a simple graph.

1.2 An undirected graph $G=(V, E) . \quad 4$

1.3 A path $P_{5}$ of order 5 and length 4.

1.4 A connected graph $G$ and a disconnected graph $H$. 6

1.5 A connected graph $G$ on five nodes. $\quad 7$

1.6 A graph $G$, a subgraph $H$, an induced subgraph $Q$, and a $\begin{array}{ll}\text { spanning subgraph } R \text {. } & 8\end{array}$

1.7 Complete graphs $K_{4}$ and $K_{6}$. 8

1.8 A cycle $C_{6} . \quad 9$

1.9 Isomorphic graphs. $\quad 9$

$\begin{array}{ll}1.10 \text { Examples of Trees. } & 10\end{array}$

1.11 A directed graph $G$. 11

1.12 Independent set on 5 nodes. 12

$1.13 \mathrm{ED}(\mathrm{P})=0.4, \mathrm{ED}(\mathrm{Q})=1, \mathrm{ED}(\mathrm{R})=1.3 . \quad 12$

$\begin{array}{lll}2.1 \text { A signed graph. } & 15\end{array}$

$2.2 X, Y, Z$ are mutual friends: balanced. 16 
$2.3 X$ and $Y$ are friends with $Z$ as a mutual enemy: balanced.

$2.4 X$ is friends with $Y$ and $Z$, but $Y$ and $Z$ are enemy to each other : unbalanced.

$2.5 X, Y$ and $Z$ are mutual enemies: unbalanced.

2.6 The labeled four-node complete graph on the left is balanced and on the right is unbalanced.

2.7 A directed graph.

2.8 A directed graph with nodes labeled with their in-degree. 22

2.9 A directed graph with nodes labeled with their out-degree. 23

2.10 Two graphs with nodes labeled with their betweenness centralities.

2.11 A tree with nodes labeled with their closeness centralities.

2.12 The node $w$ is the common out-neighbor of $u$ and $v$.

2.13 The number of common out-neighbor of $u$ and $v$ is given by $\operatorname{CON}(u, v)=3$.

2.14 CON score of $u=\mathrm{CON}(u)=5$.

2.15 In these networks we have that $\operatorname{ED}(\mathrm{R})=0.6$ and $\mathrm{ED}(\mathrm{S})=1.2 .28$

2.16 A heat map representation of dynamic competition networks according to the $\mathrm{DCH}$, where nodes closer to the center have higher closeness and CON scores. Larger nodes have higher CON scores, lower in-degree, and higher out-degree. 
The induced subgraphs (which are the circles) correspond to alliances.

3.1 Survivor: Borneo.

3.2 Survivor: China.

3.3 Survivor: Game Changer. 44

3.4 Survivor: HHH. 46

3.5 Big Brother, Season 12. 


\section{List of Tables}

1 Survivor: Borneo, Season 1.

2 Survivor: China, Season 15.

3 Survivor: Game Changer, Season 34.

4 Survivor: Heroes vs Healors vs Hustlers (HHH), Season 35.

5 Big Brother, Season 12. 47

6 Edge densities of the Alliances. $\quad 49$ 


\section{CHAPTER 1}

\section{Introduction}

\subsection{Motivation}

We are connected to each other via our social interactions, either as individuals or in large groups. A social network is a collection of agents that are connected by some particular types of social interactions such as friendship, enemies, likes or dislikes. We can observe, analyse and measure social networks with the help of observable data. Social Network Analysis (SNA) considers networks, where nodes are individual agents in the network and edges are the relationship or links between the agents. For example, we denote edges as following in Twitter, while edges represent friendship in Facebook.

We may apply SNA [17] to analyse the networks in the field of economics, computer science, political science, communication studies, geography, social psychology and also in reality shows in television. In social networks, we often consider social interaction as a positive relationship such as friendship, likeness or some type of common interest between the individuals or groups. We may also see the negative social relationship in network, which is also play an important role to find the hidden interactions between the connections. 
Bonato et al. [5], introduced the Iterated Local Transitivity (ILT) model for a social networks. The central idea of ILT model is transitivity: if $x$ is a friend of $y$, and $y$ is a friend of $z$, then $x$ is a friend of $z$. On the other hand Structural Balance Theory $[\mathbf{9}, \mathbf{1 3}]$ gives the inverse idea: if $x$ is an enemy of $y$, and $y$ is an enemy of $z$, then $x$ is a friend of $z$.

As evidence of negative social interactions, we may consider the enmity between the nations, rival gangs or competition between players in games. Another example is the stock market graph [4], where the nodes are stocks and two stocks are adjacent if they are negatively correlated.

Based on the competitive and negative interactions in a social network, we consider directed social networks of competing agents that evolve dynamically over time, where directed edges represent some kind of negative relationships between the agents in the network. As an example relevant to this thesis, a directed edge may be a vote of one contestant to other in the game show Survivor. One of our main contributions, as first described in Chapter 2, is the introduction of the Dynamic Competition Hypothesis $(\mathrm{DCH})$ to identify the alliances and top players within the dynamic competition network. We verify our hypothesis by using the voting history data of social game shows Survivor and Big Brother. 


\subsection{Introduction to Graph Theory}

In this section, we discuss some basic definitions from graph theory which we use throughout our thesis. A graph $G=(V, E)$ is a pair consisting of a node set $V=V(G)$, an edge set $E=E(G)$ consisting of pairs of nodes. Note that $E$ is taken as a multiset, as its elements may occur more than once. We write $u v$ if $u$ and $v$ form an edge, and say that $u$ and $v$ are adjacent.

A loop is an edge whose endpoints are equal. Multiple edges are edges having the same pair of endpoints. We restrict our attention to simple graphs; that is, graphs without loops and multiple edges. Further, we only consider finite graphs.
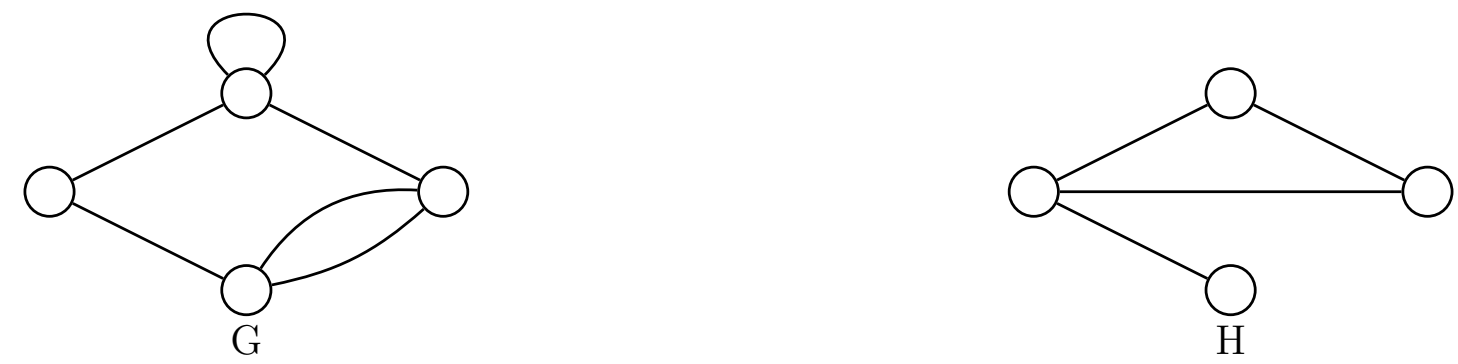

Figure 1.1. $G$ has multiple edges and a loop and $H$ is a simple graph.

The degree of a node $v$ in $G$, written $\operatorname{deg}_{G}(v)$, is the number of neighbors of $v$ in $G$. We will drop the subscript $G$ if the graph is clear from context.

The set of all nodes adjacent to a node $u$, is called the neighborhood of a node $u \in V(G)$, denoted by $N(u)$. We denote $N[u]$ be the closed neighborhood of $u$, written as $N[u]=N(u) \cup\{u\}$. We denote by $\mid V(G \mid$ 
the order of nodes $V$ in $G$; the total number of nodes in $V$ in graph $G$. We denote by $|E(G)|$ the order of edges $E$ in $G$; the total number of edges in $E$ in graph $G$. We note that

$$
|E| \leq\left(\begin{array}{c}
|V| \\
2
\end{array}\right)
$$

The degree of the node $u \in V(G)$, denoted by $\operatorname{deg}(u)$ is equal to the number of edges incident on $u$. The node $u$ is leaf or pendant if $\operatorname{deg}(u)=1$. The node $u$ is isolated if $\operatorname{deg}(u)=0$.

Now we give an example of an undirected graph $G=(V, E)$, where the nodes $V=\{a, b, c, d\}$ and edges $E=\{a b, b c, b d, d a\}$.

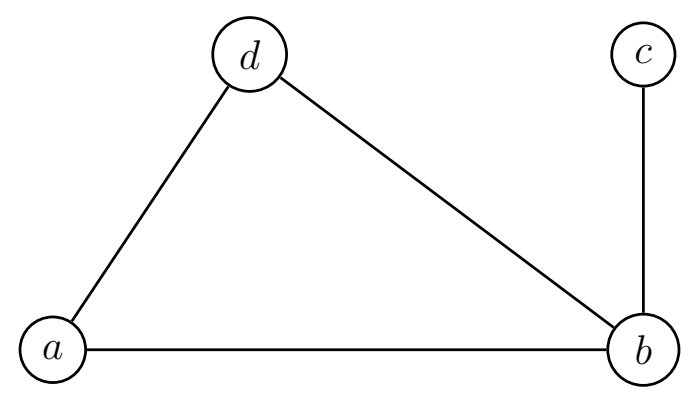

Figure 1.2. An undirected graph $G=(V, E)$.

In Figure 1.2, node $a$ is adjacent to nodes $d$ and $b$ but it is not adjacent to $c$. The neighborhood of node $a$ is the graph with two nodes $d$ and $b$ and one edge connecting $b$ and $d$. The degree of nodes are $\operatorname{deg}(a)=\operatorname{deg}(d)=2$, $\operatorname{deg}(b)=3, \operatorname{deg}(c)=1$. We note that $c$ is leaf.

The following is often referred to as the First Theorem of Graph Theory (which is included for completeness). 
THEOREM 1. [5] If $G$ is a graph, then we have that

$$
\sum_{v \in V(G)} \operatorname{deg}(v)=2|E(G)| .
$$

Proof. Let $S=\sum_{v \in V(G)} \operatorname{deg}(v)$. Notice that in $S$, we count each edge exactly twice. Thus, $S=\sum_{v \in V(G)} 2|E(G)|$.

A sequence of $n$ nodes such that each node is adjacent to the next node in the sequence, is called the path of order $n$. We denote this graph by $P_{n}$ and the length of the path is $n-1$.

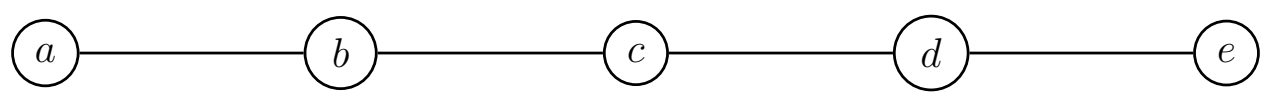

Figure 1.3. A path $P_{5}$ of order 5 and length 4 .

A walk in a graph $G=(V, E)$ is an alternating sequence

$$
W=\left\langle v_{o}, e_{1}, v_{1}, e_{2}, \ldots, v_{n-1}, e_{n}, v_{n}\right\rangle
$$

between the nodes and edges such that $e_{i}=\left(v_{i-1}, v_{i}\right)$, for all $v_{i} \in V(G)$ and $e_{i} \in E(G), i=1,2, \ldots, n$. The number of edge-steps in the walk called the length of the walk. A walk that begins and ends at the same node is said to be closed walk. A walk that begins and ends at different node called an open walk. A walk that does not pass through the same edges twice is called a trail.

A graph $G$ is said to be connected if for each distinct pair of nodes $u, v \in V(G)$ there is a path from $u$ to $v$ in graph $G$; otherwise the graph 
$G$ is disconnected. The distance from the nodes $u$ to $v$ is the length of the shortest path from $u$ to $v$ in a connected graph $G$, denoted by $d(u, v)$. If $u$ and $v$ are in different components, then $G$ is said to be disconnected such that $d(u, v)=\infty$.
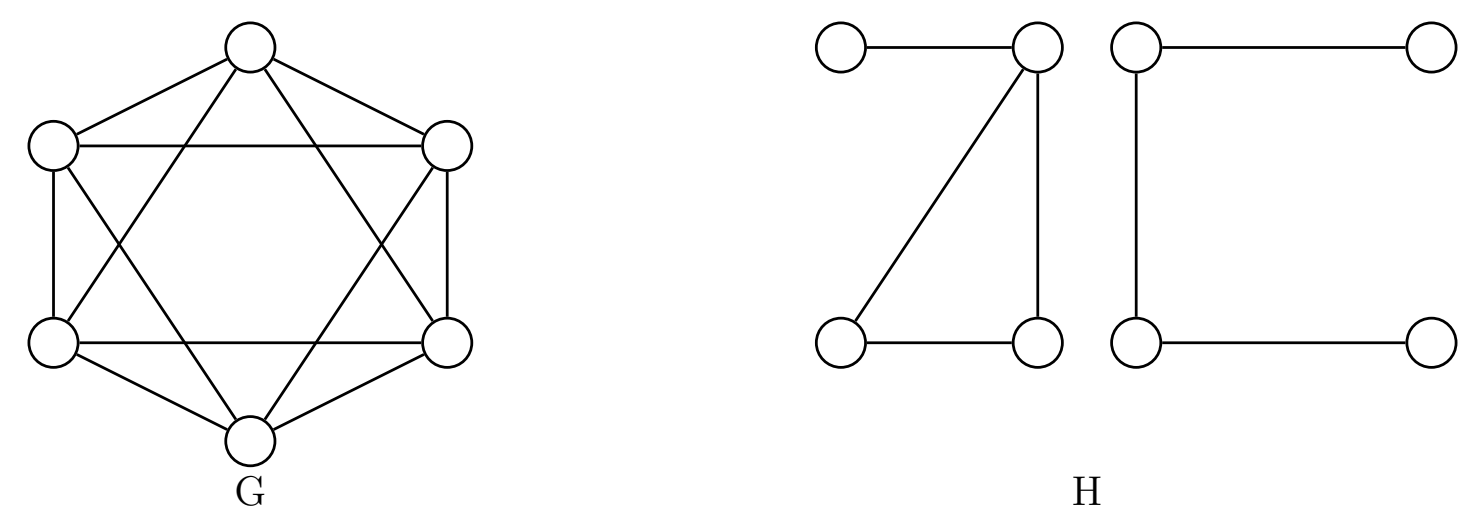

$\mathrm{H}$

Figure 1.4. A connected graph $G$ and a disconnected graph $H$.

The eccentricity of a node $v$, denoted by $e(v)$, of a connected graph $G=(V, E)$ is the maximum distance from node $v$ to any other node $u \in V(G)$; that is,

$$
e(v)=\max _{u \in V(G)}\{d(u, v)\} .
$$

The maximum eccentricity in a graph $G$ is the diameter of a graph $G$, denoted by $\operatorname{diam}(G)$; that is,

$$
\operatorname{diam}(G)=\max _{v \in V(G)}\{e(v)\}
$$


The minimum eccentricity in the graph $G$ is the radius of a graph $G$, denoted by $\operatorname{rad}(G)$; that is,

$$
\operatorname{rad}(G)=\min _{v \in V(G)}\{e(v)\}
$$

A node with minimum eccentricity in a graph $G$ is called a central node and we write

$$
e(v)=\operatorname{rad}(G)
$$

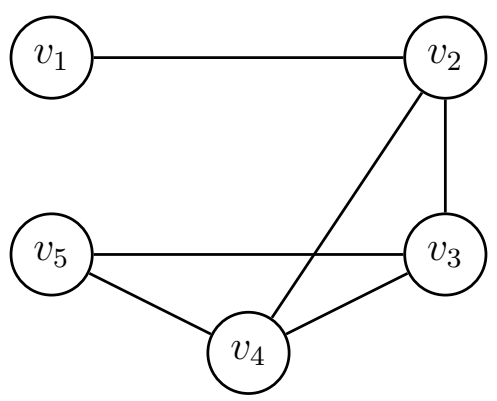

Figure 1.5. A connected graph $G$ on five nodes.

In Figure 1.5, the eccentricities of the nodes of graph $G$ are $e\left(v_{1}\right)=$ $e\left(v_{5}\right)=3$, and $e\left(v_{2}\right)=e\left(v_{3}\right)=e\left(v_{4}\right)=2$. The diameter and radius of $G$ are $\operatorname{diam}(G)=3$ and $\operatorname{rad}(G)=2$, respectively.

A graph $H$ is a subgraph of a graph $G$ when all of its nodes and edges are contained in $G$. That is $V(H) \subseteq V(G)$ and $E(H) \subseteq E(G)$, which we represent by $H \subseteq G$. A spanning subgraph $H$ of a graph $G$ is a graph in which $V(H)=V(G)$. A subgraph $H=(V(H), E(H))$ of graph $G=(V(G), E(G))$ is said to be an induced subgraph of $G$, if for all 
$u, v \in V(H)$ joined by an edge $(u, v) \in E(G)$ then $(u, v) \in V(H)$, we represent induced subgraph of $G$ by $H[G]$.
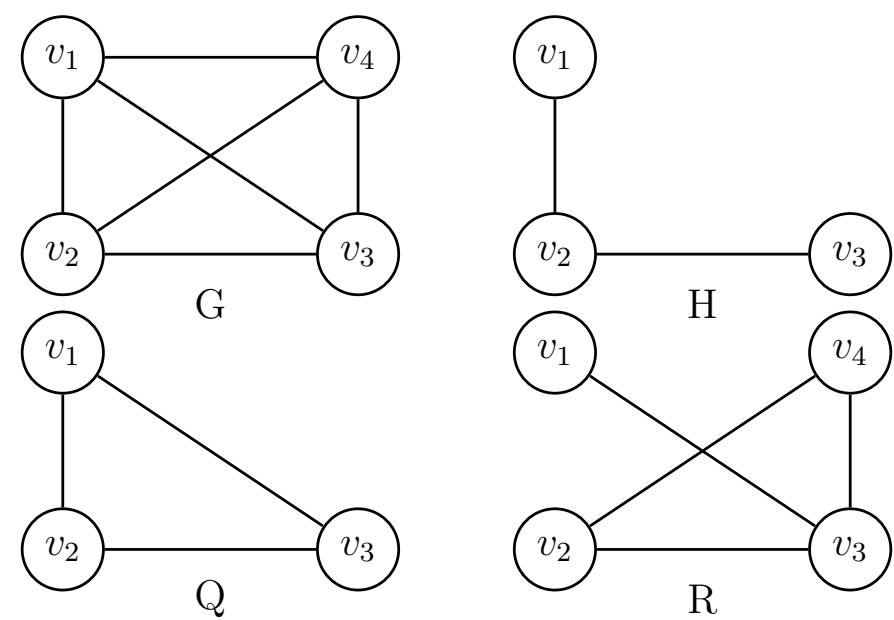

Figure 1.6. A graph $G$, a subgraph $H$, an induced subgraph $Q$, and a spanning subgraph $R$.

A graph of $n$ nodes is said to be complete or clique if all distinct nodes are joined to each other by an edge. We represent a complete graph by $K_{n}$.

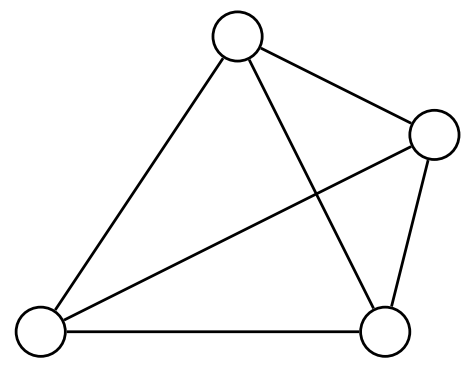

$K_{4}$

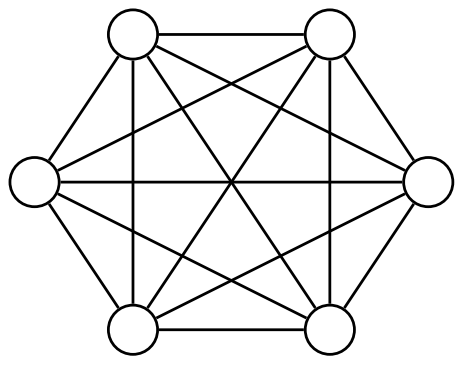

$K_{6}$

Figure 1.7. Complete graphs $K_{4}$ and $K_{6}$.

A connected graph with $n$ nodes is said to be a cycle in which the number of nodes equal to the number of edges, we represent a cycle by $C_{n}$. A graph with no cycle is said to be acyclic. 


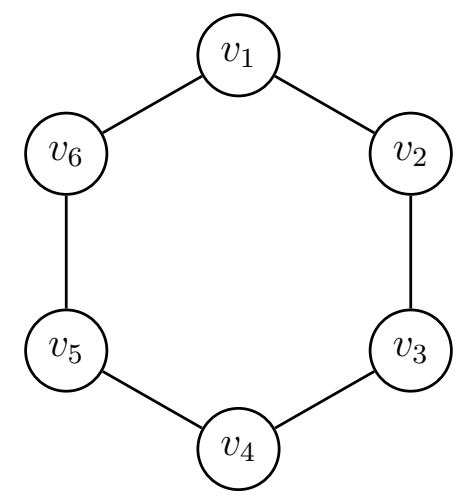

Figure 1.8. A cycle $C_{6}$.

Let $G=(V(G), E(G))$ and $H=(V(H), E(H))$ be two graphs. A homomorphism of $G$ to $H$ is a function $f: V(G) \rightarrow V(H)$ such that for all pair of nodes $x, y \in V(G), x y \in E(G)$ implies that $f(x) f(y) \in E(H)$. We denote a homomorphism from $G$ to $H$ as $G \rightarrow H$. An isomorphism is a bijective function $f: G \rightarrow H$ such that $x y$ is an edge in $G$ if and only if $f(x) f(y)$ is an edge in $H$. Note that two isomorphic graphs share all the same properties.
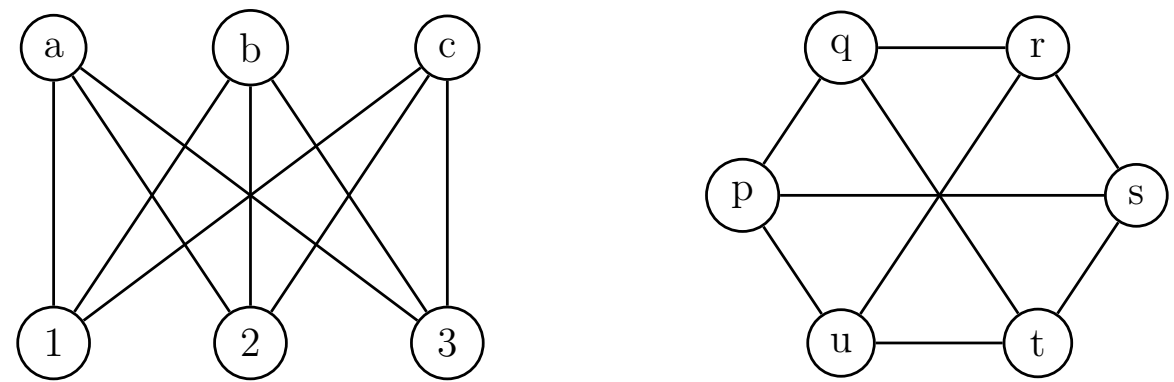

FigURE 1.9. Isomorphic graphs.

A connected graph $G=(V, E)$ that has no cycle is called a tree. A node has degree one is called leaf in a tree, whereas branches are called the edges in tree. In a graph in which all of whose connected components 
are trees said to be forest; the connected components of a graph is a subgraph in which any two nodes are connected to each other by path.
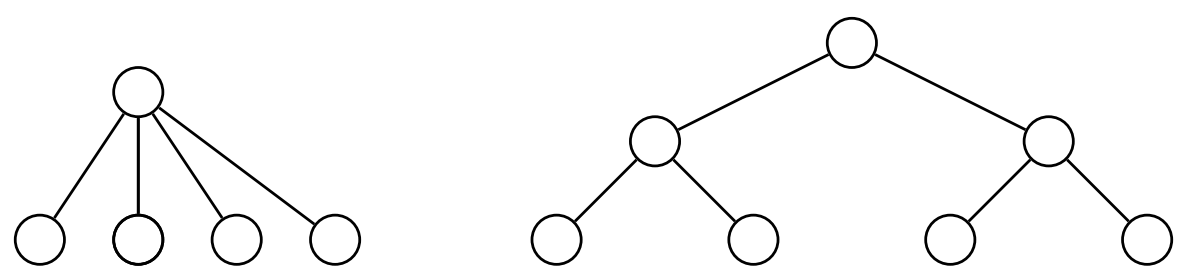

Figure 1.10. Examples of Trees.

Here we include below some equivalent definitions of a tree (which we include for completeness).

Theorem 2. [18] Given a graph $G=(V, E)$ with $|V(G)|=n$, the following are equivalent.

(a) $G$ is a tree.

(b) G has no cycles.

(c) The number of edges of $G$ is one less than the number of nodes of $G$.

(d) Every pair of nodes $u, v \in G$ is connected by unique path.

(e) Removing any edge from $G$ gives a graph which is not connected.

We now define directed graphs. A graph $G=(V, E)$ is said to be directed graph or digraph if the edge set $e_{i} \in E(G)$ consists of ordered node pairs $(u, v)$, for all $u, v \in V(G)$. The edge $e$ is said to be directed edge from $u$ to $v$ if it is associated with the pair $(u, v)$ of nodes. The number of edges incoming to a node called in-degree, denoted by $\operatorname{deg}^{-}(u)$, 
where $u$ is a node. The number of edges outgoing from a node called outdegree, denoted by $\operatorname{deg}^{+}(u)$. A node has zero in-degree called source node, where a node has zero out-degree called sink node. We have the following theorem, which is part of folklore (which is included for completeness).

TheOREM 3. For every digraph $G$,

$$
\sum_{v \in V(G)} \operatorname{deg}^{+}(v)=\sum_{v \in V(G)} \operatorname{deg}^{-}(v)
$$

Proof. For every digraph $G=(V(G), E(G))$, an edge has two ends with one end of the edge adding 1 to the out-degree of some $v \in V(G)$, while the other end of the edge adding 1 to the in-degree of the some $v \in V(G)$.

In Figure 1.11, we find an example of a directed graph $G$ with nodes $v_{1}, v_{2}, v_{3} \in V(G), \operatorname{deg}^{-}\left(v_{1}\right)=1, \operatorname{deg}^{-}\left(v_{3}\right)=2$ and $\operatorname{deg}^{-}\left(v_{2}\right)=0$, where $\operatorname{deg}^{+}\left(v_{1}\right)=1, \operatorname{deg}^{+}\left(v_{2}\right)=2$ and $\operatorname{deg}^{+}\left(v_{3}\right)=0$. We see that node $v_{2}$ is a source node and node $v_{3}$ is sink node.

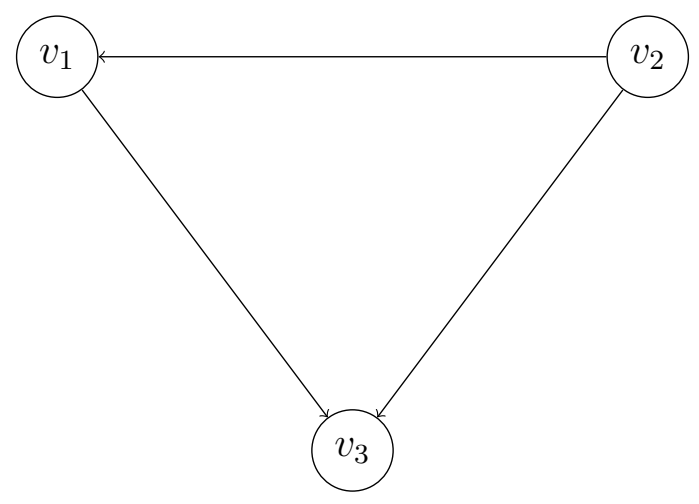

Figure 1.11. A directed graph $G$. 
A digraph $G$ is said to be weighted graph if all of its nodes or edges assigned a weight. An edge-weighted graph has weight on its edges and node weighted graph has weight on its nodes.

A set of nodes $W \subseteq V(G)$ with no directed edges within $W$, then the set $W$ is an independent set.

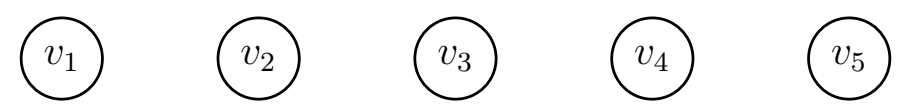

Figure 1.12. Independent set on 5 nodes.

An independent set $W$ of a digraph $G$ is said to be maximum if $G$ has no independent set $W^{\prime}$ such that $\left|W^{\prime}\right|>|W|$. The independence number of $G$, denoted by $\alpha(G)$, is the number of nodes in a maximum independent set of $G$. The edge density of the set of nodes $S$ is the ratio

$$
E D(S)=\frac{|E(S)|}{\left(\begin{array}{c}
|S| \\
2
\end{array}\right)} .
$$

See Figure 1.13 for an example.

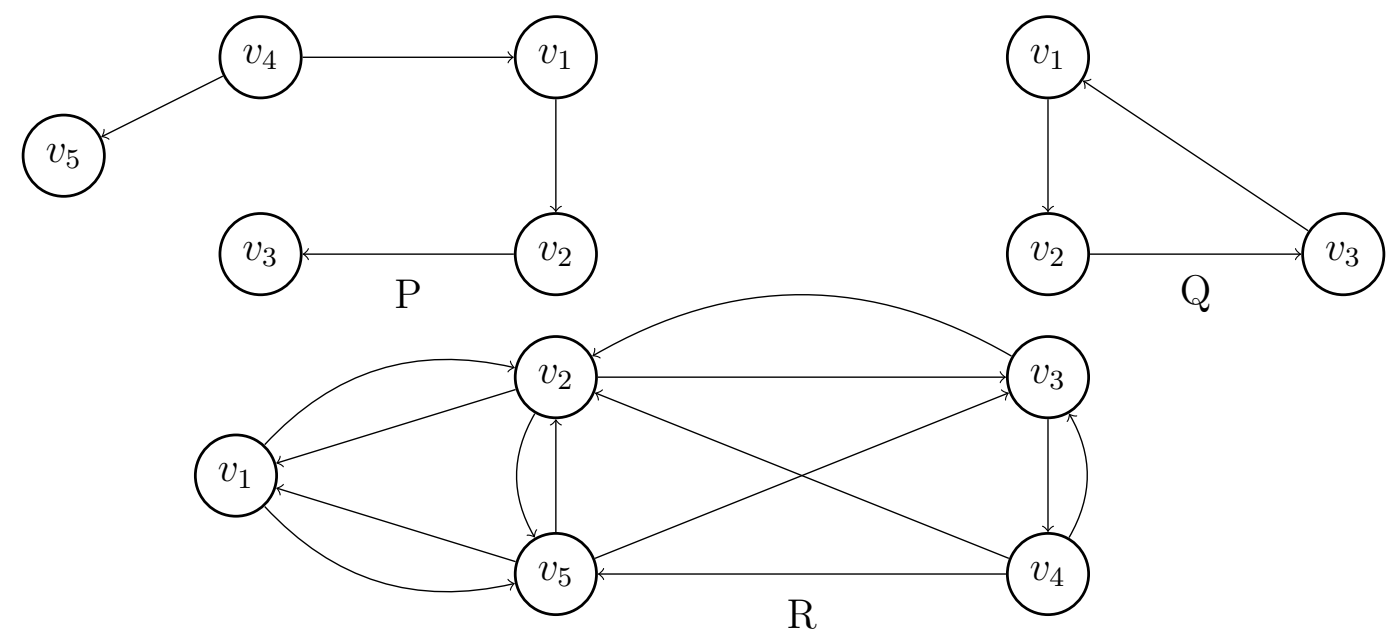

Figure 1.13. $\mathrm{ED}(\mathrm{P})=0.4, \mathrm{ED}(\mathrm{Q})=1, \mathrm{ED}(\mathrm{R})=1.3$. 


\subsection{Summary of the Thesis}

In this chapter, we discussed the basic definitions and notation from graph theory, citing various properties and theorems. In Chapter 2, we will define centrality metrics on graphs, with the aim of determining important nodes in social networks. We will discuss structural balance theory and alliances in social networks. We will introduce and study the properties of dynamic competition networks. We formulate the Dynamic Competition Hypothesis that detects the leaders and measures the relative strength of the alliances in the social network.

In Chapter 3, we will present voting history data of four seasons from U.S. Survivor and one data set from Big Brother. We will analyse and verify these data sets by applying network metrics to validate the Dynamic Competition Hypothesis. We will find that the Dynamic Competition Hypothesis exactly predicts the alliances, top players and finalists in the game shows.

In the last chapter, we summarize and discuss our results. We will present open problems derived from our analysis and describe how the Dynamic Competition Hypothesis can be applied in other types of complex networks. 


\section{CHAPTER 2}

\section{The Dynamic Competition Hypothesis}

In this chapter, we introduce and discuss structural balance theory. We define a model of positive and negative relationships in a complete graph. We discuss balanced and unbalanced graphs in signed social networks with examples. We define standard centrality metrics in network science that will be used to state the Dynamic Competition Hypothesis in Chapter 3. We introduce dynamic competition networks in real-world social networks. We formulate the Dynamic Competition Hypothesis with the help of graph theoretic tools to identify the alliances and leaders in a social network.

\subsection{Structural Balance Theory}

We discuss here the basic model of positive and negative relationships modeled by a complete graph. Suppose we have a social network representing a set of people, in which everyone knows everyone; that is, there is an edge joining each pair of nodes. We label each edge with either a positive sign $(+)$ or a negative sign $(-)$. A positive edge between two nodes denotes a positive relationship (such as friendship or an alliance) and a negative edge between two nodes denotes a negative relationship 
(such as hatred or competition). Such a labeled graph is called a signed network.

In signed social networks, there is the concept of balanced and unbalanced cycles. A balanced cycle is defined as a cycle where the product of all the signs are positive. Balanced graphs represent a group of people who are unlikely to change their opinions of the other people in their group. In contrast, the unbalanced graphs represent a group of people who are very likely to change their opinions of the other people in their group.

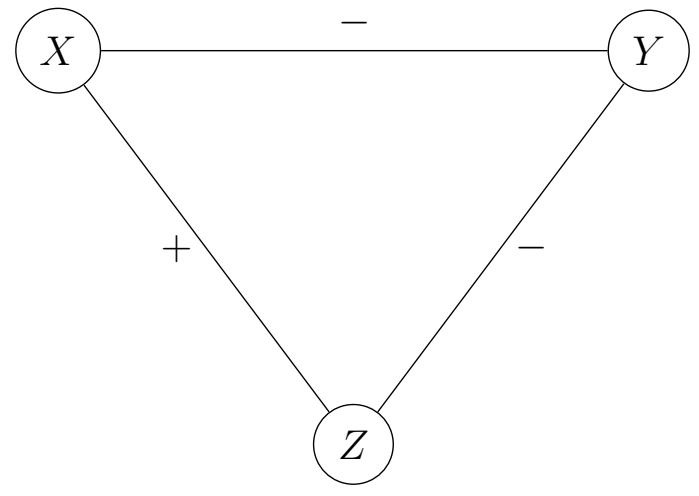

Figure 2.1. A signed graph.

The principles underlying structural balance are based on theories in social psychology from the work of Heider in the 1940s [14], and the theory was generalized and extended to the language of graphs with the work of Cartwright and Harary in the 1950s; see $[\mathbf{7}, \mathbf{8}, \mathbf{1 2}]$. The critical idea of structural balance theory is that if we consider any two people in isolation, then the edge between them can be + or - ; that is, they are either friends or enemies. But when we look at sets of three people at a 
time, there are four distinct ways to label the three edges among three people with +'s and -'s. We can differentiate these four possibilities as follows.

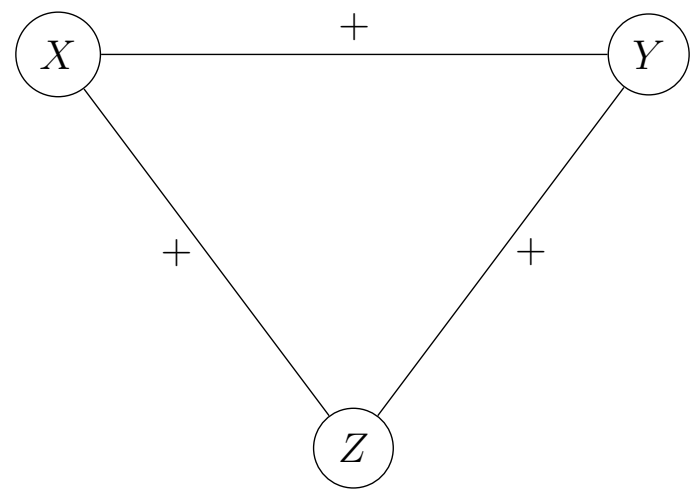

Figure 2.2. $X, Y, Z$ are mutual friends: balanced.

In Figure 2.2, it is a natural situation if in a given set of three people $X, Y$ and $Z$, they have three positive signs among them: it corresponds to three people who are mutual friends.

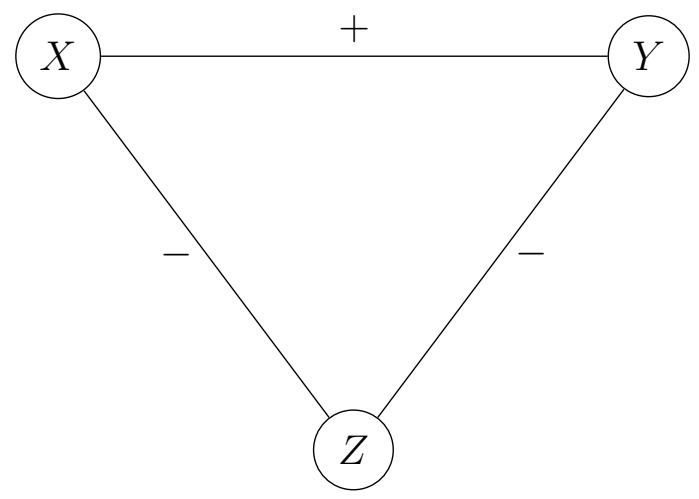

Figure 2.3. $X$ and $Y$ are friends with $Z$ as a mutual enemy: balanced.

In Figure 2.3, it is also natural if a single positive sign and two negative signs in the relations among the three people. It means that two of the three are friends, and they have a mutual enemy in the third. 


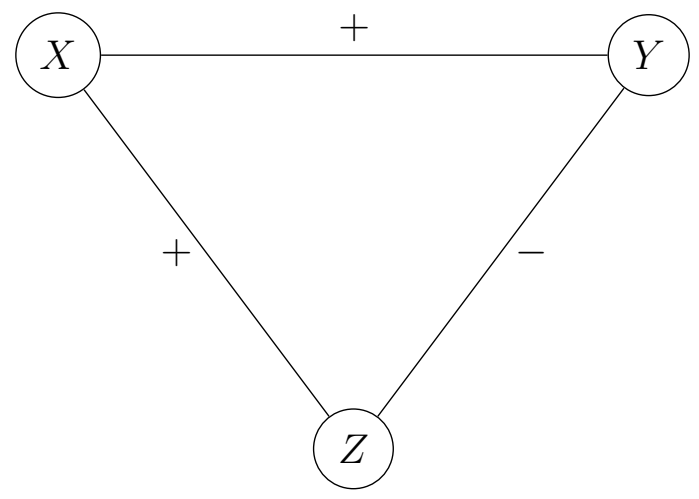

Figure 2.4. $X$ is friends with $Y$ and $Z$, but $Y$ and $Z$ are enemy to each other : unbalanced.

In Figure 2.4, a triangle with two positive signs and one negative sign corresponds to a person $X$ who is a friends with each of $Y$ and $Z$, but $Y$ and $Z$ are not friend with each other. This type of labeling shows some kind of psychological stress or instability between the relationships. In this situation, there would be implicit forces pushing $X$ to try to get $Y$ and $Z$ to become friends (changing the $Y Z$ edge label to + ); or else for $X$ to side with one of $Y$ or $Z$ against the other (changing one of the edge labels incident with $X$ to a - ).

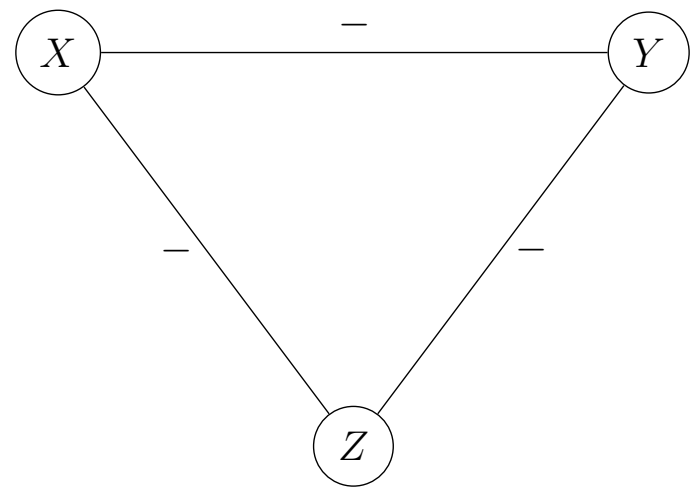

Figure 2.5. $X, Y$ and $Z$ are mutual enemies: unbalanced. 
In Figure 2.5, there are also sources of instability in an arrangement where $X, Y$ and $Z$ are mutual enemies. In this situation, there would be forces motivating two of the three people to become partners against the third (changing one of the three edge labels to $\mathrm{a}+$ ).

Due to the above reasons in the labeled triangles, we indicate the triangles who have one or three + labels as balanced and triangles with zero or two + labels as unbalanced. In structural balance theory, each labeled triangle must have one or three positive edges.

We may view unbalanced triangles as sources of stress or psychological dissonance, which strive to minimize in their personal relationships. Hence, they will be less abundant in real social settings than balanced triangles.

We next generalize our definition of structural balance of complete graphs for groups of three nodes into an arbitrary number of nodes with edges labeled by +'s and -'s. In particular, the labeled complete graph is balanced if it obeys the following property:

Structural Balance Property: For every set of three nodes, if we consider the three edges connecting them, either all three of these edges are labeled + , or else exactly one of them is labeled + .

We consider an example of two labeled networks in Figure 2.6. We see that the labeled four-node complete graph on the left consists of two 
groups of friends $X, Y$ and $Z, R$, with negative relations between people in different groups. It is balanced because each set of three nodes satisfies the Structural Balance Property. The labeled four-node complete graph on the right is not balanced because there are exactly two edges labeled + between three nodes $X, Y, Z$ and the triangle $Y, Z, R$ violates the Structural Balance Property.

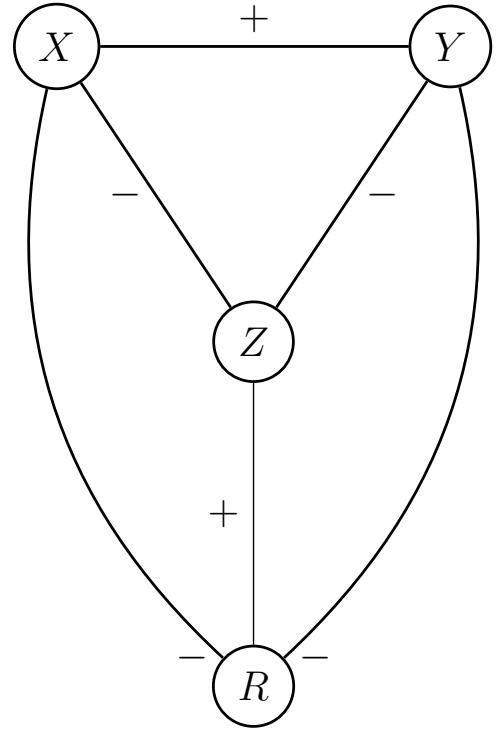

Balanced

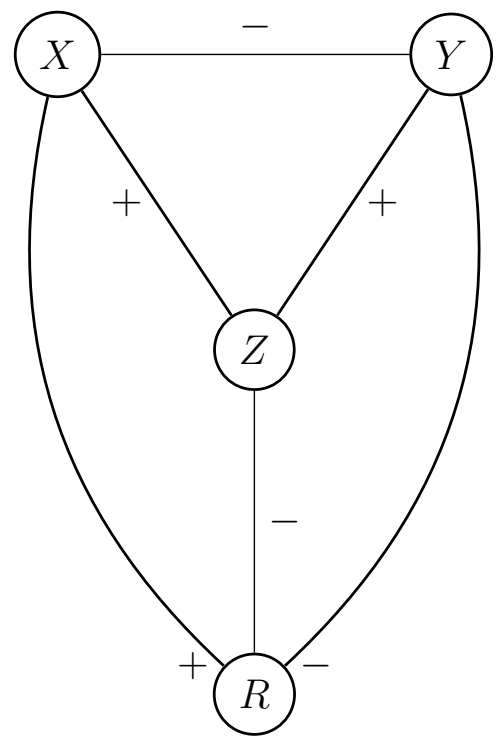

Not banlaced

Figure 2.6. The labeled four-node complete graph on the left is balanced and on the right is unbalanced.

We see that there are only two basic ways for structural balance network to occur: everyone likes each other (see Figure 2.2), or the network consists of two groups of mutual friends with complete antagonism between the groups. This fact was originally discovered by Drown Cartwright and Frank Harary in the 1950s. 
TheOREM 4. $[\mathbf{8}, \mathbf{1 2}]$ If a labeled complete graph is balanced, then either all pairs of nodes are friends, or else the nodes can be divided into two groups, $X$ and $Y$, such that every pair of people in $X$ like each other, every pair of people in $Y$ like other, and everyone in $X$ is the enemy of everyone in $Y$.

\subsection{Centrality metrics in networks}

In this section, we present some graph-theoretic terminology, and we will define standard centrality metrics in network science that we will use later. For example, by using various centrality metrics, we can find the most influential nodes.

A network can be represented by its matrix called the adjacency matrix. We define the adjacency matrix of a graph $G=(V(G), E(G))$ with $n$ nodes by the $n \times n$ matrix with entries $A_{i, j}$ given by:

$$
A_{i, j}= \begin{cases}1 & \text { if }\left(v_{i}, v_{j}\right) \in E(G) \\ 0 & \text { otherwise }\end{cases}
$$

for all $v_{i}, v_{j} \in V(G)$.

We consider an example to find the adjacency matrix of the following graph, whose nodes are $v_{1}, v_{2}, v_{3}, v_{4}, v_{5}$ and $v_{6}$, respectively. 


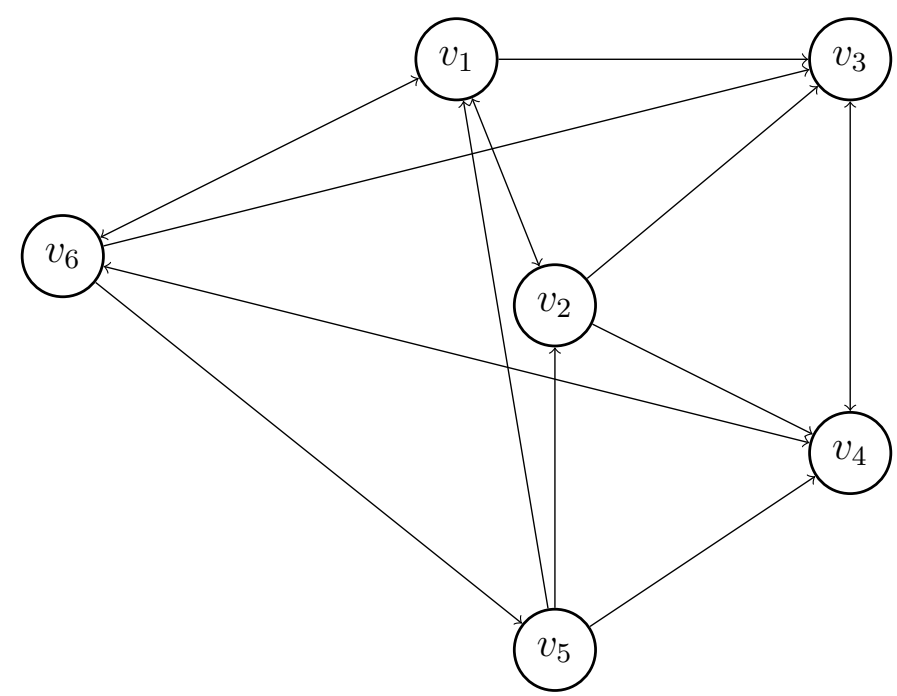

Figure 2.7. A directed graph.

The adjacency matrix of the directed graph in Figure 2.7 is the following:

$$
A_{i, j}=\left[\begin{array}{llllll}
0 & 1 & 1 & 0 & 0 & 1 \\
1 & 0 & 1 & 1 & 0 & 0 \\
0 & 0 & 0 & 1 & 0 & 0 \\
0 & 0 & 1 & 0 & 0 & 1 \\
1 & 1 & 0 & 1 & 0 & 0 \\
1 & 0 & 1 & 1 & 1 & 0
\end{array}\right]
$$

Degree centrality simply measures the degrees of nodes. Degree may be interpreted in terms of the immediate risk of node catching whatever is flowing through the network (such as a virus, influence, or information). For a directed network, we define two types of degree centrality: in-degree and out-degree. 
Let $A_{i, j}$ be the adjacency matrix of a directed graph. The in-degree centrality $x_{i}$ of node $i$ is equal to:

$$
x_{i}=\sum_{k} A_{k, i} .
$$

See Figure 2.8 for an example.

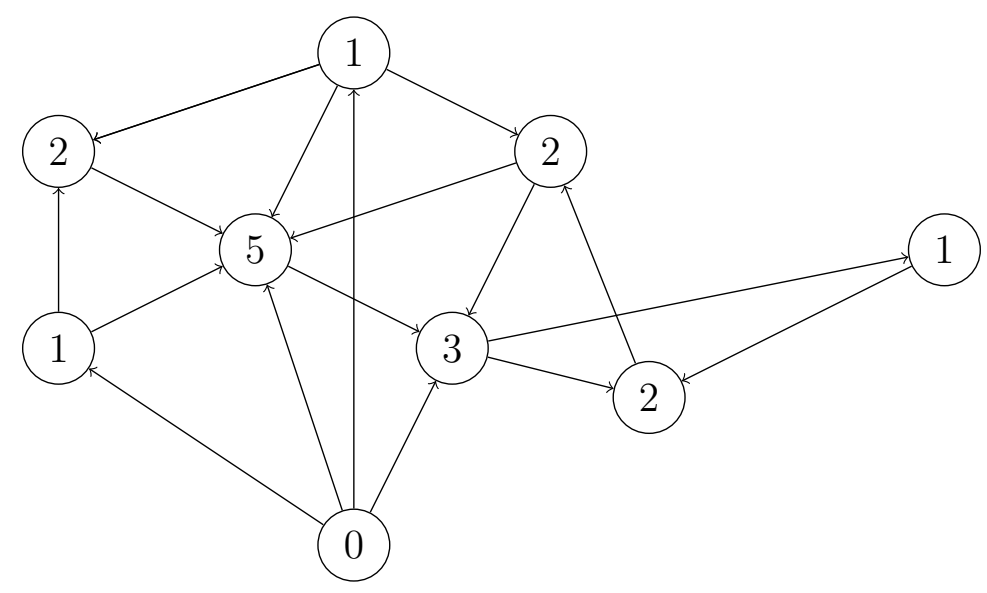

FIgURE 2.8. A directed graph with nodes labeled with their in-degree.

The out-degree centrality $y_{i}$ of node $i$ is equal to:

$$
y_{i}=\sum_{k} A_{i, k} .
$$

See Figure 2.9 for an example.

We consider degree centrality as a local metric since it only considers neighbors of nodes. For example, in an on-line social network such as Facebook, degrees measure the relative popularity of users.

Betweenness centrality is an important statistical property of a network. This is applied in many real-world problems, such as finding influential people in a social network, finding crucial hubs in a computer 


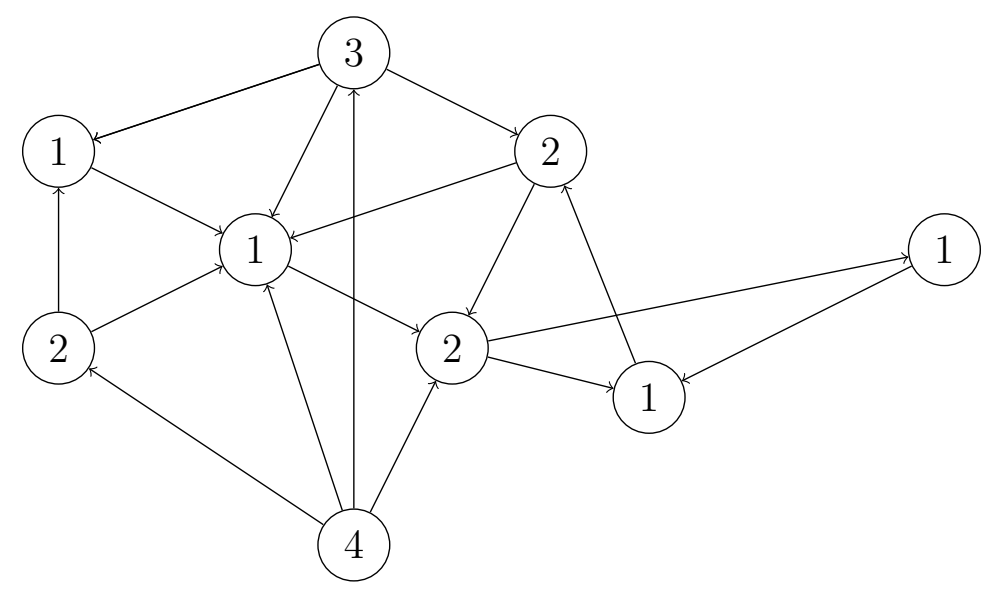

Figure 2.9. A directed graph with nodes labeled with their out-degree.

network, or finding border crossing points which have the most trade flow. See $[10,11]$.

The betweenness of a node $u$ in a graph $G=(V, E)$ is computed as follows:

(1) For each pair of nodes $(v, w)$, compute the shortest paths between them.

(2) For each pair of nodes $(v, w)$, determine the fraction of shortest paths that pass through the node $u$.

(3) Sum this fraction over all pairs of nodes $(v, w)$.

More formally, we define the betweenness centrality of $u$ by

$$
B(u)=\sum_{v, w \in V(G) \backslash\{u\}} \sigma_{v w}(u) / \sigma_{v w}
$$

where $\sigma_{v w}$ denote the total number of shortest one-way, directed paths between $v, w$ and $\sigma_{v w}(u)$ denote the number of shortest one way, directed 
paths between $v$ and $w$ that pass through the node $u$. If there does not exist any shortest path between $v$ and $w$, then we have that $B(u)=0$.
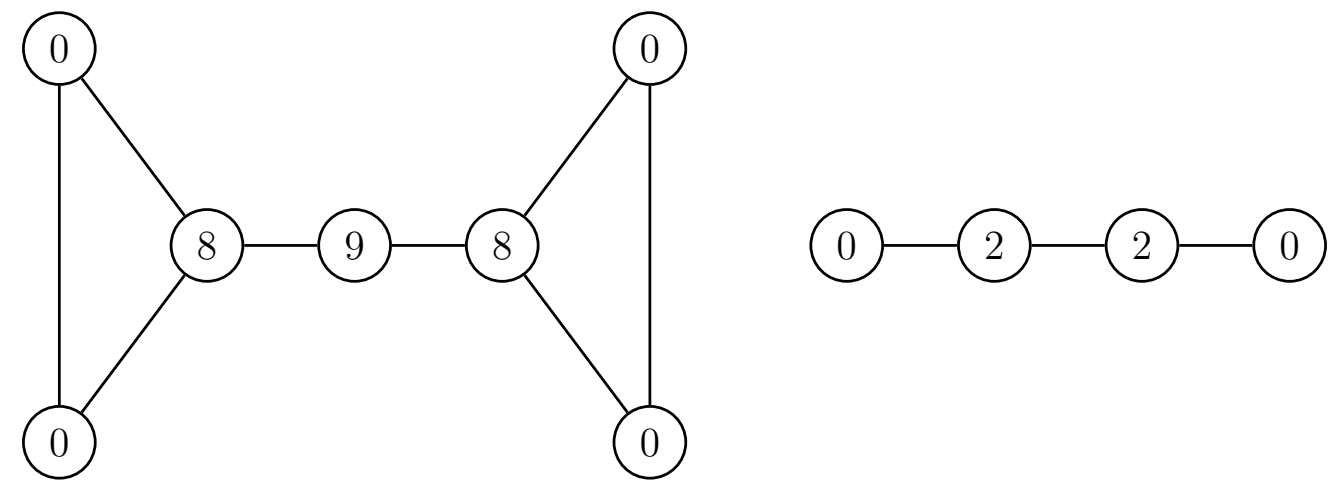

Figure 2.10. Two graphs with nodes labeled with their betweenness centralities.

In connected graphs there is a natural distance metric between all pairs of nodes, defined by the length of their shortest paths. The farness of a node $u$ is defined as the sum of its distances from all other nodes in a graph. The farness of node $u$ is defined by

$$
F(u)=\sum_{v \in V(G)} d(u, v)
$$

Closeness centrality indicates how long it will take for information from a given node to reach other nodes in the network. We describe the closeness centrality as the reciprocal of the farness. For a connected digraph $G=(V, E)$ and a node $v \in G$, we define closeness centrality of $u$ by

$$
C(u)=\left(\sum_{v \in V(G) \backslash\{u\}} d(u, v)\right)^{-1}
$$


where $d(u, v)$ is the distance measured by one-way, directed paths from $u$ to $v$. Thus, the more central a node is the lower its total distance from all other nodes. Note that taking distances from or to all other nodes is irrelevant in undirected graphs, whereas in directed graphs distances to a node are considered a more meaningful measure of centrality; see [2].

Both closeness and betweenness are well-studied centrality measures for complex networks; see [6]. For example, centrality of sports networks is often used to rank teams; see [15].

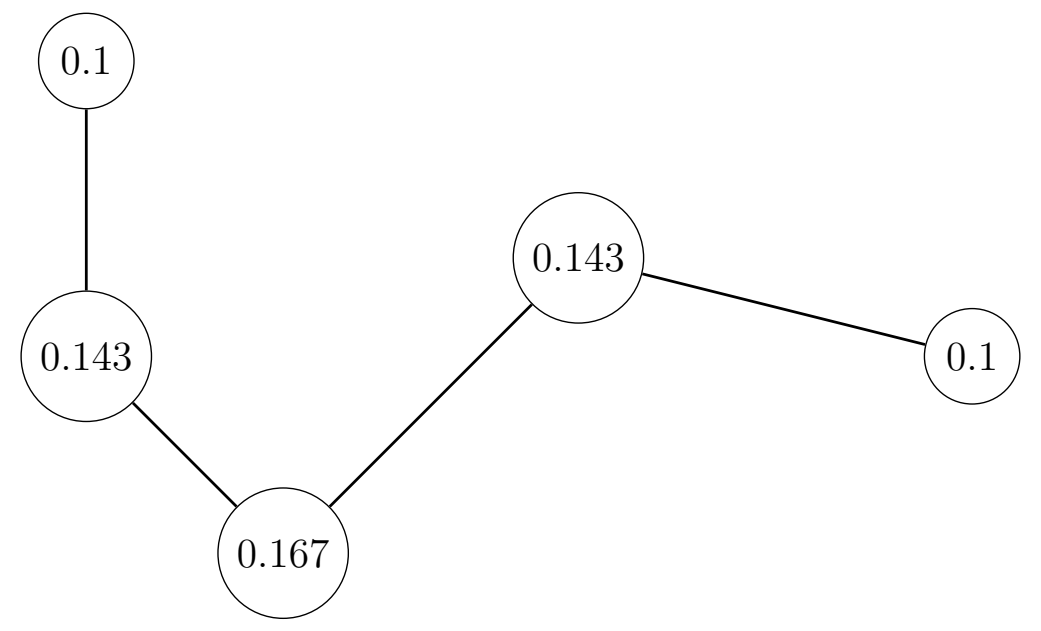

Figure 2.11. A tree with nodes labeled with their closeness centralities.

In Chapter 3, we will apply network science in the social game show Survivor. Given the nature of the voting network in Survivor, we also consider the number of common out-neighbors as a key metric which define as follows.

For nodes $u, v$, and $w$, we say that $w$ is a common out-neighbor of $u$ and $v$ if $(u, w)$ and $(v, w)$ are directed edges. 


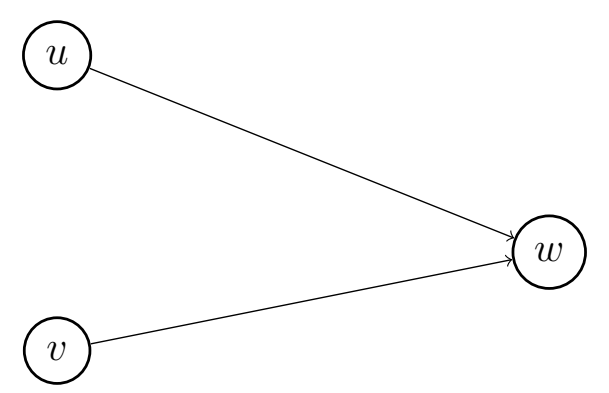

Figure 2.12. The node $w$ is the common out-neighbor of $u$ and $v$.

For a pair of distinct nodes $u, v$, we define $\operatorname{CON}(u, v)$ to be the number of common out-neighbors of $u$ and $v$.

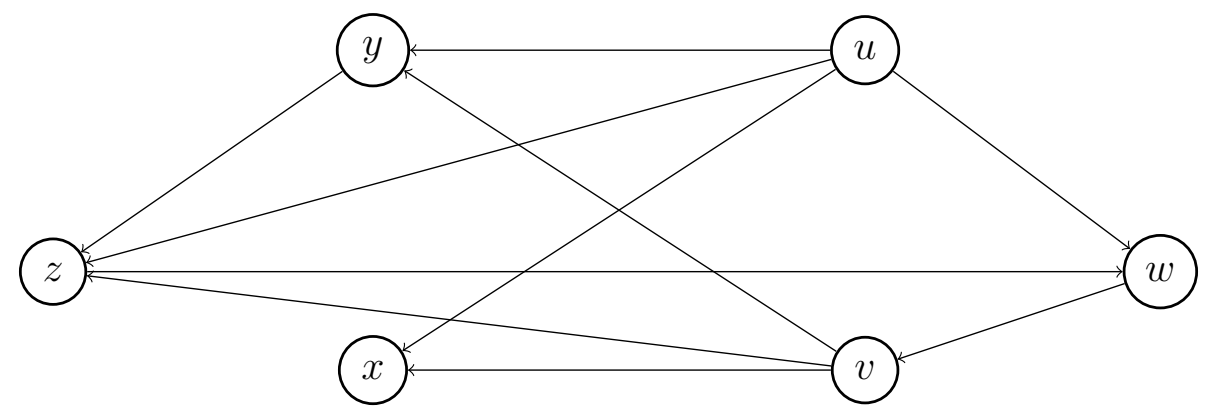

FigURE 2.13. The number of common out-neighbor of $u$ and $v$ is given by $\operatorname{CON}(u, v)=3$.

For a fixed node $u$, define

$$
\operatorname{CON}(u)=\sum_{v \in V(G)} \operatorname{CON}(u, v)
$$

We call $\mathrm{CON}(u)$ the $C O N$ score of $u$.

For a set of nodes $S$ with at least two nodes, we define

$$
\operatorname{CON}(S)=\sum_{u, v \in S} \operatorname{CON}(u, v)
$$

We note that $\operatorname{CON}(S)$ is a non-negative integer. 


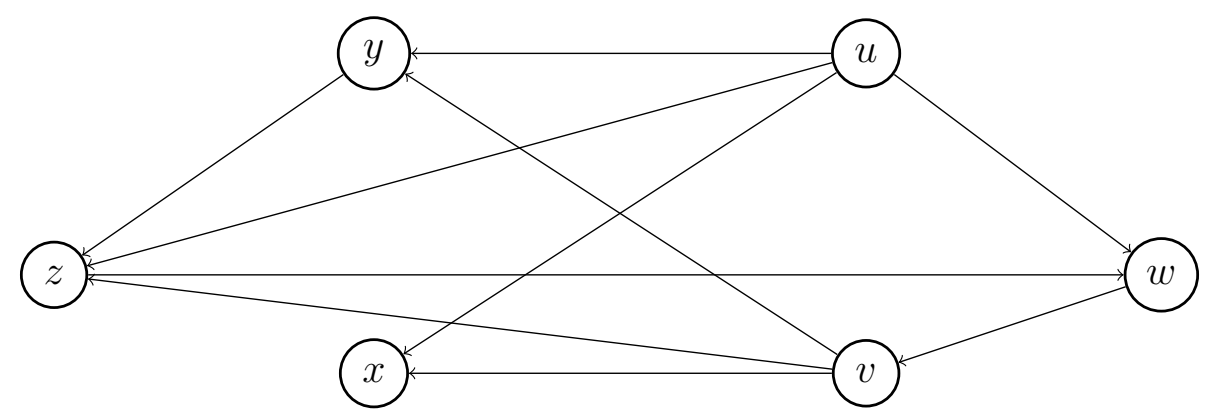

FIGURE 2.14. CON score of $u=\mathrm{CON}(u)=5$.

For a set $S$ of nodes, we denote its edge density by $E D(S)$, and compare this to that of an independent set. We observe that $E D(S)$ may be greater than 1 as there may be multiple edges in the digraphs as we defined in Chapter 1 (see Figure 1.13).

If $E D(S) \leq \epsilon$, then a set $S$ is said to be $\epsilon$-near independent, where $\epsilon$ is a non-negative real number. We measure the relative density of sets of nodes by the parameter $\epsilon$. If the set $S$ is $\epsilon$-near independent for some positive value of $\epsilon$ then $S$ is called near independent. Generally, in applications, we take $\epsilon$ to be small. The value of $\epsilon$ will often be heuristically determined in a real-world networks by considering a ranking of subsets by their edge density (we highlight that every set of nodes is $\epsilon$-near independent for a suitably chosen $\epsilon$ ). We note that independent sets are near independent by choosing the value of $\epsilon=0$.

In Figure 2.15, we consider two sets of nodes $R$ and $S$ where we have that $E D(R)=0.6$ and $E D(S)=1.2$, respectively. We choose $\epsilon_{1}$ and $\epsilon_{2}$ such that $\epsilon_{1}=0.6$ and $\epsilon_{2}=1.3$. We see that $E D(R) \leq \epsilon_{1}$ and 


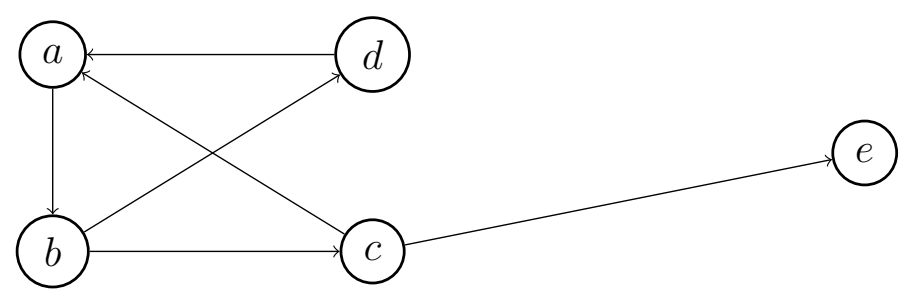

$\mathrm{R}$

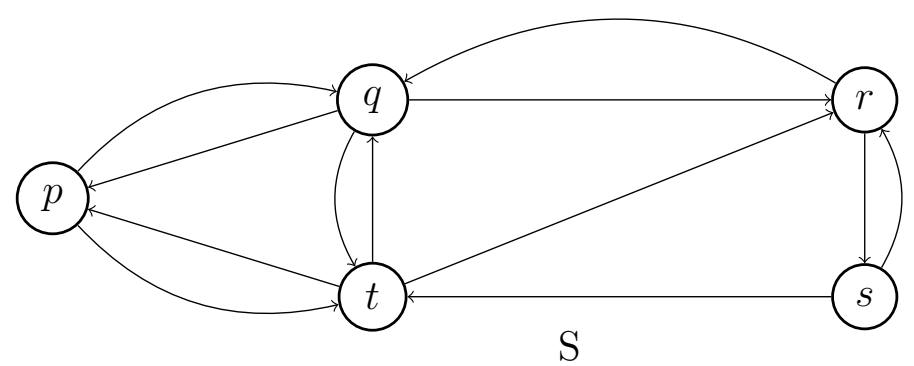

Figure 2.15. In these networks we have that $\mathrm{ED}(\mathrm{R})=0.6$ and $\mathrm{ED}(\mathrm{S})=1.2$.

$E D(S) \leq \epsilon_{2}$, then we say that set $R$ is $\epsilon_{1}$-near independent and set $S$ is $\epsilon_{2}$-near independent.

\subsection{Dynamic Competition Networks}

We consider a network $G$ in which nodes represent agents, and there may be directed edges from nodes $u$ and $v$. We say $G$ is a competition network if agent $u$ is in competition with agent $v$. A directed edge may correspond to some kind of negative social interaction from nodes $u$ to $v$; that is, a directed edge $(u, v)$ may represent a vote against $v$. Note that competition networks need not be tournaments, which are directed graphs isomorphic to those where every pair of nodes has exactly one directed edge between them.

A competition network is said to be dynamic competition network if directed edges are added over discrete time-steps. For example, in the 
game show Survivor, contestants cast votes against each other, and a directed edge may be a vote of one contestant to other; see Chapter 3. We consider another example from real-world networks, where nodes represents nation states and edges correspond to conflicts between them. Dynamic competition networks may have multiple edges. In our thesis, we focus on the underlying structure of social networks of competitors which we call a dynamic competition network. Specifically, we focus on the networks arising on the American television series Survivor and Big Brother. Before we describe our hypothesis, we discuss some important definitions which we use in our hypothesis and whole thesis.

Alliance means a bond or connection between families, states, individuals, or parties. In the real-world network, we find alliances in many ways, for example:

(1) Companies with common economic interests.

(2) People who unite by relationship or friendship.

(3) Political parties.

(4) Friendly nation states.

We define alliances in the social game show Survivor, as groups of contestants who work together to vote off contestants outside the alliance. In particular, alliances are induced subgraphs of dynamic competition networks. In the case of strong alliances, members of an alliance are less likely to vote for each other. 
Persons that hold a dominant or superior position in the network are called leaders and are able to exercise a high degree of control or influence over others; that is, edges emanating from leaders may influence edge creation in other agents. Leaders may be the winner in the game show Survivor or may be the non-winning players with a strong hold on the results of the game. Our main goal is to apply network science to help determine alliances and leaders in dynamic competition networks arising in social networks.

\subsection{The Hypothesis}

Structural Balance Theory posits relationships as either positive or negative, and in our approach, we focus on negative relationships. We apply network science to identify the alliance and top players within a dynamic competition network such as Survivor. Players in Survivor are represented by nodes and there is a directed edge from player $x$ to player $y$ if $x$ votes for $y$. The directed edges in Survivor correspond to competition or rivalry between the players, and so correspond to negative relationships. Directed edges are added over discrete time steps in dynamic competition network.

We indicate the top players in Survivor with their in-degree. Players who have low in-degree to be considered as top players. Alliances have low edge densities and are near independent. We note that the top 
players have high CON scores and we consider them as a leader within the alliance.

An important measure of top players is the closeness. Players who are centrally located in the network have highest closeness. We discover that the closeness appears as a reliable predictor in Survivor.

The Dynamic Competition Hypothesis (or DCH) asserts that dynamic competition networks arising from a social network satisfy the the following four properties.

(1) Alliances are near independent sets.

(2) Strong alliances have low edge density.

(3) Members of an alliance with high CON scores are more likely leaders.

(4) Leaders exhibit high closeness, high CON scores, low in-degree, and high out-degree.

We see the visualization of DCH in Figure 2.16. Now we observe that the above four properties jointly support each other. When we detect an alliance from property (1) then we are able to compute the strength of an alliance relative to other alliances by using property (2). We can separate leaders within alliances by applying property (3). Specifically, we anticipate leaders to be in alliances because of having their eminent local control in the alliances. We consider property (4) is independent of 


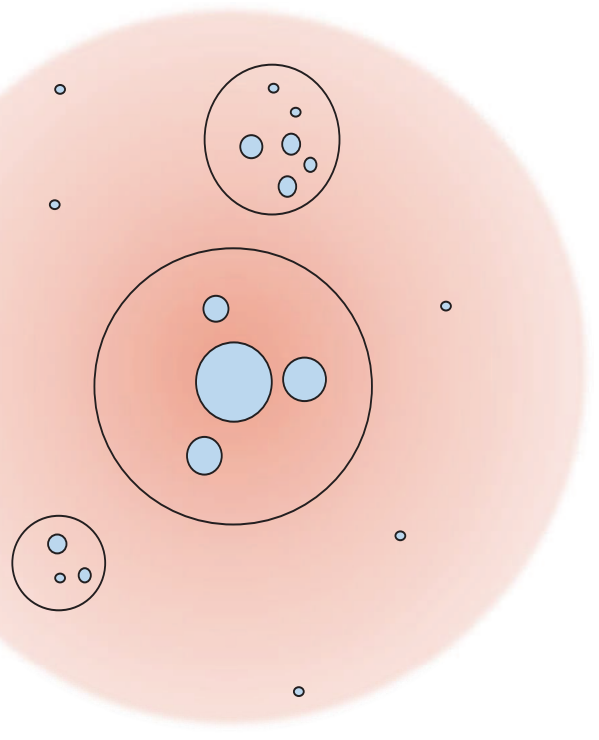

FiguRE 2.16. A heat map representation of dynamic competition networks according to the $\mathrm{DCH}$, where nodes closer to the center have higher closeness and CON scores. Larger nodes have higher CON scores, lower in-degree, and higher out-degree. The induced subgraphs (which are the circles) correspond to alliances.

alliances and we determine leaders through global metrics of the network; that is, we measure closeness centrality.

In Chapter 3, we will test the Dynamic Competition Hypothesis (DCH) with historical voting data from four seasons of the U.S. television social game shows Survivor and one season from Big Brother. 


\section{CHAPTER 3}

\section{Data and Methods}

In this chapter, we provide background on the U.S. based social game shows Survivor and Big Brother. We concentrate on four seasons from Survivor and one season from Big Brother, in detail. We examine the voting data of these seasons by using tools from network science. We observe that the Dynamic Competition Hypothesis (DCH) predicts the formation of alliances and finalists with a high degree of accuracy. As indicated in Chapter 2, the Dynamic Competition Hypothesis (or DCH) asserts that the dynamic competition networks arising from a social network satisfy the following four properties (which we restate for convenience):

(1) Alliances are near independent sets.

(2) Strong alliances have low edge density.

(3) Alliance members who have high CON scores are leaders.

(4) Leaders reveal high closeness, high CON scores, low in-degree, and high out-degree.

\subsection{Survivor}

Survivor is a reality-based competition television show. Survivor is an example of a social game, where social interactions establish the direction 
of the play and winners. We observe that there are versions of Survivor in many countries. In our thesis, we consider the U.S. version of Survivor.

In Survivor, a group of contestants called survivors are marooned in an isolated location, usually with a tropical climate. Survivors are forced to provide food, water, and shelter for themselves with insufficient help from the outside world. Survivors are split into two or more tribes, which cohabitate and work together. As a tribe, the survivors must endure the elements, build shelter, look for water, and scrounge for food and other requirements for the whole period of the game, which is generally 39 days in the U.S. version.

In particular, in the first half of the game, the tribes compete in challenges, some for rewards of food, shelter, or luxury items, while others are for immunity, preventing the winning tribe from having to go to the tribal council. The losing tribe goes to tribal council, where they vote out one of their own tribesman to eliminate them from the game.

In the second half of the game, the tribes are merged into a single tribe, and the remaining survivors compete for individual rewards and immunity. At subsequent tribal councils, eliminated players start to form the jury, who sit in on all subsequent tribal councils but they do not participate in the voting. When there are a small number of remaining survivors who are finalists; that is, only two or three, they attend the final tribal council, where the jury is given the opportunity to ask them 
questions. After this, the jury members vote in favor of one of them to become the Sole Survivor who receives a cash prize of one million dollars.

\subsection{Big Brother}

Big Brother is a reality-based competition television game show in which a group of contestants are called HouseGuests. HouseGuests cohabitate in a custom-built house under constant video surveillance. While in the house, the contestants are isolated from the outside world; that is, no phone, television, Internet, magazines, newspapers, or have no communication with those not in the house. This rule could be broken in the case of medical injury and any family emergency. The HouseGuests are free to leave from the game but they are not allowed to entry back into the house. HouseGuests could be expelled from the house if they break the rules of the game.

Each week, the HouseGuests compete for the title of Head of Household. Head of Household nominates two HouseGuests for eviction. The HouseGuests vote to evict one of them. The HouseGuests who has the most votes is evicted from the game. Once only two HouseGuests remain, the members of the Jury cast their votes for who should win the series. The winner receives a cash prize of half a million dollars.

Various twists have been introduced during the seasons in both Survivor and Big Brother to keep the players vigilant and to avoid players relying a similar game plan used in preceding seasons. For example, in 
Survivor, these twists include tribal switches, seasons starting with more than two tribes, the ability to exile a player from a tribe for a short time, hidden immunity idols that players can use to save themselves or others at tribal council or special voting powers which can be used to influence the result at tribal council. As a disclaimer, our use of network analysis is insensitive to these twists.

\subsection{Social Network Analysis tools}

Social network analysis tools are used to identify, analyze, visualize or simulate nodes (agents) and edges (relationships) from various types of input data including mathematical models of social networks. There are several tools available for analysis of social networks. We selected the software Gephi [1], which is freely available for use and can handle large graph sizes. Gephi is a tool to explore and understands graphs. We used Gephi in our thesis for computing centrality metrics and the visualizations of dynamic competition graphs.

We have gathered data from Survivor Wiki [16] and Big Brother Wiki [3]. Data includes the information on contestants, their voting history and tribes, and catalogues of alliances.

We now present the visualizations of dynamic competition networks for Survivor and Big Brother. We discuss four seasons from Survivor; that is, Borneo, China, Game Changer, and Heroes vs Healers vs Hustlers $(\mathrm{HHH})$, respectively. The reason for choosing these seasons is because 
Borneo and HHH introduce the new players and Game Changer includes the returning contestants from the preceding seasons. In addition, several new twists and changes have been made in these seasons. We also discuss a data from Season 12 of Big Brother. We note that in both Survivor and Big Brother, we choose data when all the players cast their votes against each other. We also provide the tables which contains the summary of the relevant network statistics. In the tables below, we list the contestants in the order which they were voted out from the game. The winner of the game is the first entry of the table and the others are placed by when they were voted out from the game. We analyse the voting history data from Survivor and Big Brother by using Gephi in which we check whether the DCH predicts the top players (which may be the finalist) and the emergence of the alliances. We use the abbreviations ID, OD, C, CON, and B which stand for in-degree, out-degree, closeness centrality, CON-score, and betweenness centrality, respectively.

\subsection{Borneo}

We discuss the first season of Survivor set in Borneo. Borneo consisted of thirty-nine days of gameplay with sixteen competitors. The sixteen contestants were split into two tribes of eight: Tagi and Pagong. Both Tagi and Pagong coped equally in challenges but diverged in organisational structure. Three contestants from Pagong: Colleen, Jenna, Gervase formed an alliance, called the Barbeque alliance, whereas the four 
contestants Richard, Rudy, Susan, and Kelly from other tribe formed an alliance, called the Tagi alliance. The Tagi alliance voted as a block to ensure their safety. The Tagi alliance took advantage of the lack of voting strategy of other contestants. There was also a dispute in the Tagi alliance as Kelly was considered untrustworthy. When ten players remained, the contestants merged into one tribe. After merging in one tribe, the Tagi alliance successfully used their votes to eliminate the other contestants.

When only the Tagi alliance members remained in the game, we see that the close allies Richard and Rudy voted for Susan, and Susan and Kelly voted for Richard, so the vote ended with tie. After the revote, Susan was eliminated because Kelly switched her vote from Richard to Susan. Kelly also voted for Rudy after winning her last immunity challenge. Richard and Kelly were the finalists and Richard won the game by $4-3$.

In Table 1, we see that Richard is the the Sole Survivor of the season. We note that Richard has one of the highest closeness and CON scores. Rudy and Susan have higher scores and closeness but high in-degree as compared to Richard. We see that Kelly won four consecutive immunity challenges near the end of the game and was ineligible for elimination. Since Kelly voted for both Rudy and Susan, we note that this was the decisive factor for her not winning the finale. 
TABle 1. Survivor: Borneo, Season 1.

\begin{tabular}{|l|l|l|l|c|l|c|}
\hline Name & Tribe & ID & OD & C & CON & B \\
\hline \hline Richard & Tagi & 6 & 10 & 0.737 & 42 & 28.7 \\
\hline Kelly & Tagi & 0 & 12 & 0.682 & 34 & 0 \\
\hline Rudy & Tagi & 8 & 11 & 0.778 & 45 & 36.483 \\
\hline Susan & Tagi & 7 & 10 & 0.778 & 44 & 16.467 \\
\hline Sean & Tagi & 9 & 9 & 0.7 & 38 & 17.917 \\
\hline Colleen & Pagong & 7 & 8 & 0.636 & 29 & 33.067 \\
\hline Gervaise & Pagong & 6 & 7 & 0.636 & 31 & 8.583 \\
\hline Jenna & Pagong & 11 & 6 & 0.583 & 27 & 27.85 \\
\hline Greg & Pagong & 6 & 5 & 0.412 & 15 & 4.833 \\
\hline Gretchen & Pagong & 4 & 4 & 0.56 & 17 & 7.233 \\
\hline Joel & Pagong & 4 & 3 & 0.412 & 17 & 1 \\
\hline Dirk & Tagi & 4 & 3 & 0.5 & 12 & 1.317 \\
\hline Ramona & Pagong & 6 & 2 & 0.412 & 10 & 17.733 \\
\hline Stacey & Tagi & 6 & 2 & 0.452 & 4 & 1.733 \\
\hline B.B. & Pagong & 6 & 1 & 0.298 & 5 & 0.333 \\
\hline Sonja & Tagi & 4 & 1 & 0.452 & 4 & 0.75 \\
\hline
\end{tabular}

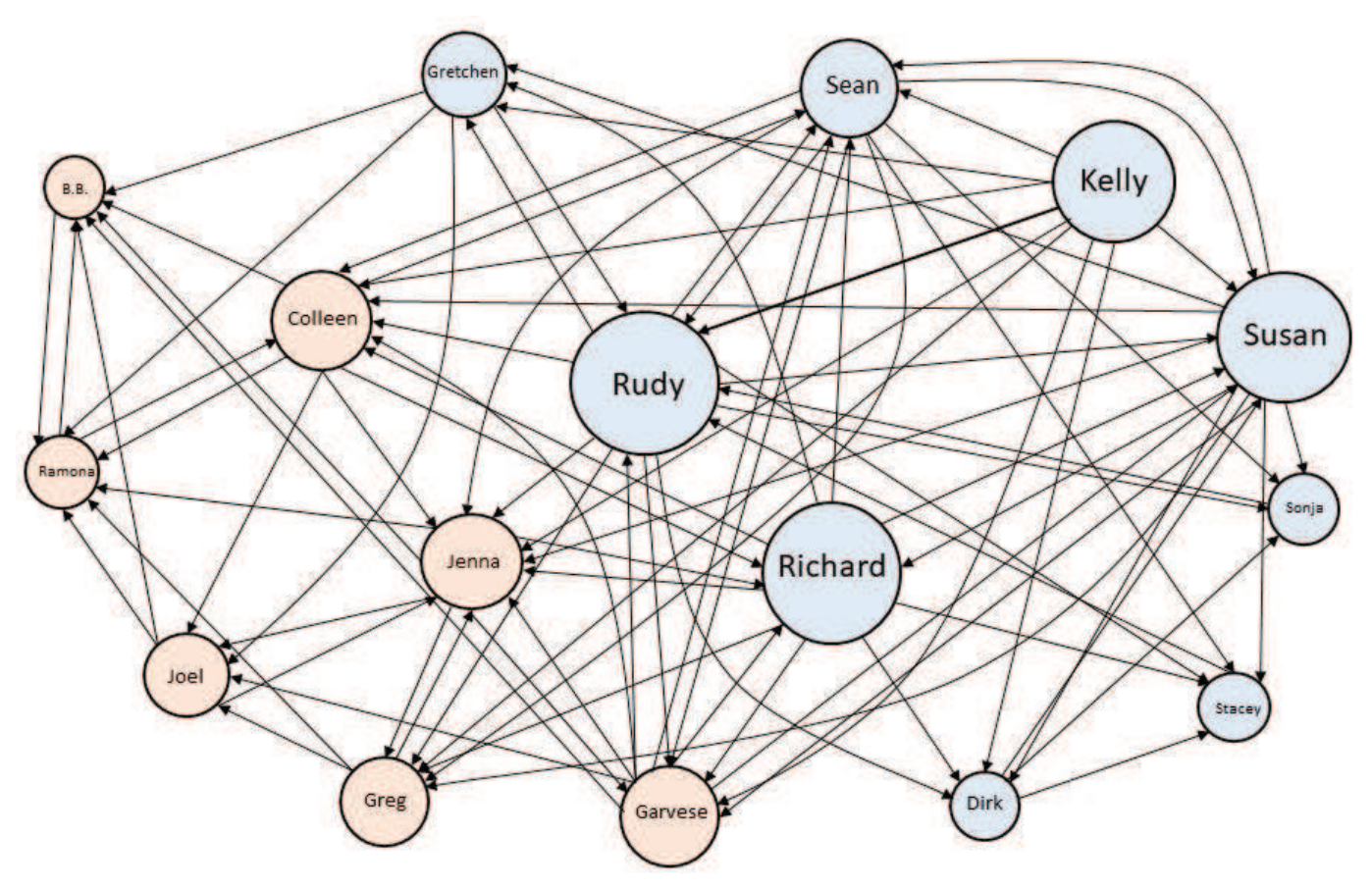

Figure 3.1. Survivor: Borneo. 
We note that comparing betweenness of the players is vague as a predictor of leaders. For example, the betweenness of Richard, Kelly, and Rudy are 28.7, 0 , and 36.5, respectively. One of the reason behind this is that the leaders tend to have lower in-degree, which may reduce the number of paths traversing through them. In the other seasons of Survivor, we do not consider, therefore, betweenness scores.

\subsection{China}

We next move to Survivor: China, which is the fifteenth season of U.S. reality television show. The reason to choose China is because it represents a sample after the game became more familiar to audiences, where contestants better understood the mechanics of the game.

Two tribes, Fei Long and Zhan Hu, were predetermined prior to the start of the game. Two alliances were formed in the tribes. Seven contestants formed the Fie Long alliance: Todd, Courtney, Amanda, Aaron, Denise, James, and Frost and three contestants Pie-Gee, Erik, and Jaime formed the Zhan $H u$ alliance.

The tribes merged in one tribe when ten contestants remained in the game, with the advantage of given to the Fei Long alliance (which comprised six contestants in the new tribe). After the elimination of two of the Fie Long's contestants, the remaining Fei Long members held on to their lead, and Todd, Amanda, and Courtney's alliance held strong. 
Todd, Amanda, and Courtney went to the final tribal council. Todd won the game with four votes to Courtney's two and Amanda's one.

In Survivor: China, we note that in Table 2, Todd is the Sole Survivor. From the table, we note that Todd has highest closeness and high CON scores. Courtney and Amanda appear also as leaders based on their CON scores.

TABle 2. Survivor: China, Season 15.

\begin{tabular}{|l|l|l|l|c|l|}
\hline Name & Tribe & ID & OD & C & CON \\
\hline \hline Todd & Fei Long & 5 & 9 & 0.765 & 49 \\
\hline Courtney & Fei Long & 0 & 9 & 0.667 & 39 \\
\hline Amanda & Fei Long & 0 & 9 & 0.737 & 49 \\
\hline Denise & Fei Long & 3 & 9 & 0.722 & 40 \\
\hline Peih-Gee & Zhan Hu & 8 & 10 & 0.722 & 41 \\
\hline Erik & Zhan Hu & 5 & 9 & 0.722 & 41 \\
\hline James & Fei Long & 9 & 6 & 0.591 & 31 \\
\hline Frosti & Zhan Hu & 7 & 7 & 0.65 & 39 \\
\hline Jean-Robert & Fei Long & 12 & 4 & 0.5 & 23 \\
\hline Jaime & Zhan Hu & 7 & 5 & 0.481 & 26 \\
\hline Sherea & Zhan Hu & 6 & 4 & 0.448 & 24 \\
\hline Aaron & Fei Long & 3 & 2 & 0.406 & 12 \\
\hline Dave & Zhan Hu & 6 & 3 & 0.382 & 11 \\
\hline Leslie & Fei Long & 6 & 1 & 0.342 & 9 \\
\hline Ashley & Zhan Hu & 8 & 2 & 0.464 & 10 \\
\hline Chicken & Zhan Hu & 5 & 1 & 0.333 & 6 \\
\hline
\end{tabular}




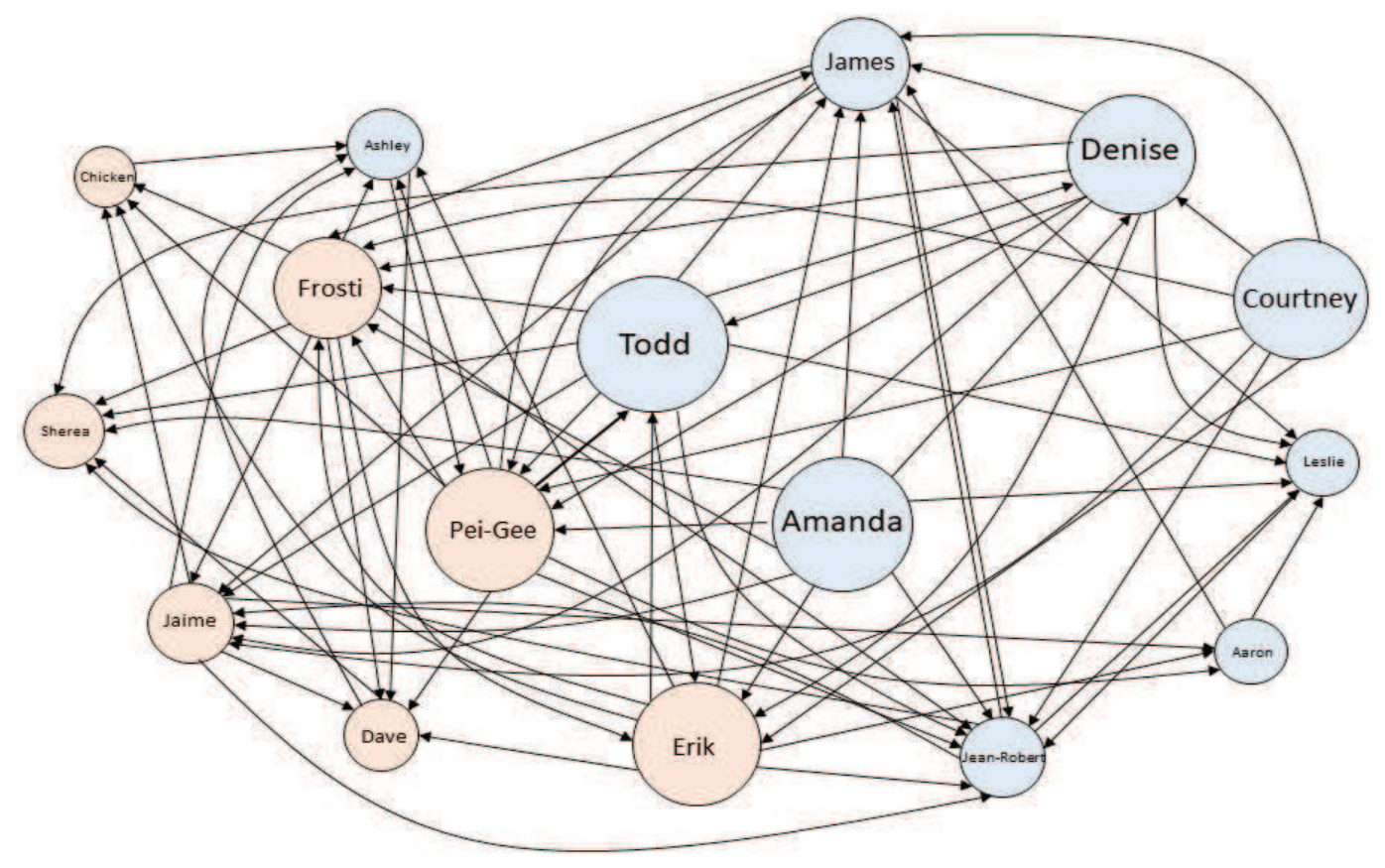

Figure 3.2. Survivor: China.

\subsection{Game Changers}

We next turn to Survivor: Game Changers, as the third-to-last season of the show. The twenty players were initially divided into two tribes: Mana and Nuku. The Nuku fared much better in challenges and the Nuku members were able to eliminate most of the members from the Mana tribe. When the tribes merged, two main factions appeared: Power Six alliance led by Sierra and Brad and Tavva alliance led by Andrea and Cirie, with Sarah navigating between the two groups to eliminate members from each. However, Sarah settled with Tavva to eliminate the other alliance members. Sarah betrayed Tavva and then aligned with Brad and Troyzan. Sarah, Brad, and Troyzan used a series of immunities and advantages to secure the majority and reach the end of 
the game together. There were ten members in the jury and Sarah won the game by taking seven votes with Brad's three.

In Table 3, We see that the Sole Survivor Sarah had higher CON scores and closeness than Brad and Troyzan. We note that Tai and Aubry had higher closeness than Sarah, Brad and Troyzan and they also had the high CON scores than both Brad and Troyzan. We note that Tai and Aubry had high in-degrees than Sarah, Brad and Troyzan which likely disadvantaged them to be in the final three.

TABle 3. Survivor: Game Changer, Season 34.

\begin{tabular}{|l|l|l|l|c|l|}
\hline Name & Tribe & ID & OD & C & CON \\
\hline \hline Sarah & Nuku & 3 & 13 & 0.692 & 64 \\
\hline Brad & Nuku & 2 & 12 & 0.643 & 49 \\
\hline Troyzan & Mana & 2 & 12 & 0.643 & 55 \\
\hline Tai & Nuku & 12 & 13 & 0.72 & 56 \\
\hline Aubry & Mana & 9 & 13 & 0.72 & 61 \\
\hline Cirie & Nuku & 0 & 8 & 0.613 & 45 \\
\hline Michaela & Mana & 11 & 11 & 0.643 & 51 \\
\hline Andrea & Nuku & 14 & 8 & 0.581 & 39 \\
\hline Sierra & Nuku & 15 & 7 & 0.581 & 34 \\
\hline Zeke & Nuku & 11 & 6 & 0.6 & 39 \\
\hline Debbie & Nuku & 6 & 7 & 0.545 & 32 \\
\hline Ozzy & Nuku & 7 & 4 & 0.5 & 22 \\
\hline Hali & Mana & 8 & 5 & 0.474 & 28 \\
\hline Jeff & Mana & 6 & 5 & 0.529 & 33 \\
\hline Sandra & Mana & 5 & 5 & 0.581 & 34 \\
\hline JT & Nuku & 3 & 2 & 0.45 & 18 \\
\hline Malcom & Mana & 5 & 3 & 0.439 & 24 \\
\hline Caleb & Mana & 5 & 3 & 0.4 & 21 \\
\hline Tony & Mana & 7 & 2 & 0.439 & 15 \\
\hline Ciera & Mana & 9 & 1 & 0.4 & 8 \\
\hline
\end{tabular}




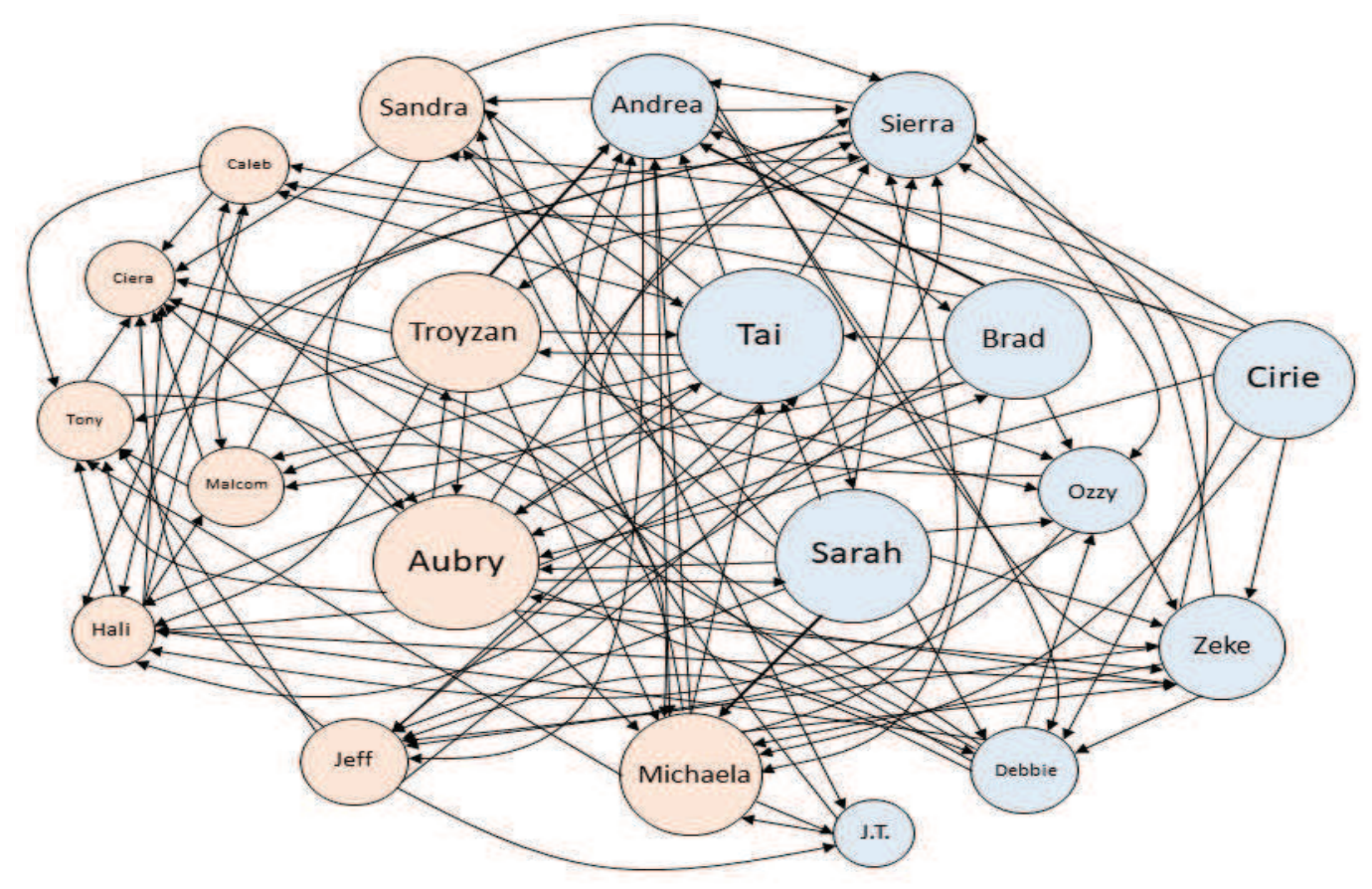

Figure 3.3. Survivor: Game Changer.

\subsection{Heroes vs Healers vs Hustlers (HHH)}

We now turn to the second-last season of Survivor: Heroes vs Healers vs Hustlers $(\mathrm{HHH})$ which is the 35 th season. This season featured 18 new players divided into three tribes based on a dominant perceived trait: with Heroes representing courage, Healers representing compassion, and Hustlers representing tenacity. The 18 players were initially divided into three tribes: Levu (Heroes), Soko (Healers) and Yawa (Hustlers). Ryan from the Hustlers tribe found a Super Immunity Idol that was only valid at the first Tribal Council. As his tribe won immunity, he sent the Idol to Chrissy who was a member of the losing Heroes tribe. Ryan and Chrissy both became dominant players on their tribes, forming strong alliances 
with Devon and Ben, respectively. The Healers tribe went undefeated, while the Heroes lost one member and the Hustlers lost two.

When the tribes were switched, Ryan and Chrissy aligned on the new Soko tribe, working to eliminate the Healers and their allies. Three alliances were formed after the tribes merged in one tribe: that is, healers, The Round Table, and Final Four alliance. Ryan and Chrissy brought their former Heroes and Hustlers tribemates together against the Healers. When Chrissy won the final immunity challenge, she had to grant one castaway additional immunity, which would force the remaining two to compete against each other in a fire-making challenge to determine the third finalist. She chose to save Ryan. Ben defeated Devon in the firemaking challenge to join Chrissy and Ryan in the finals. At the final tribal council, Ben won five jury votes, with Chrissy winning two votes, and Ryan winning one. Ben was awarded the Sole Survivor of the season.

In Table 4, we see that Ryan and Devon had the highest overall closeness of 0.708 and highest overall CON scores of 47 and 55, respectively followed by Chrissy whose closeness and CON score of 0.68 and 44, respectively. However, Ben, the Sole Survivor, had lower CON score, closeness, and high in-degree than the other finalists; that is, 41, 0.63, and 11, respectively. Ben secured his place in the final three by successfully playing three consecutive hidden immunity idols. 
TABLE 4. Survivor: Heroes vs Healors vs Hustlers (HHH), Season 35.

\begin{tabular}{|l|l|l|l|c|l|}
\hline Name & Tribe & ID & OD & C & CON \\
\hline \hline Ben & Levu & 11 & 11 & 0.63 & 41 \\
\hline Chrissy & Levu & 7 & 13 & 0.68 & 44 \\
\hline Ryan & Yawa & 2 & 14 & 0.708 & 47 \\
\hline Devon & Yawa & 2 & 11 & 0.708 & 55 \\
\hline Mike & Soko & 9 & 9 & 0.63 & 37 \\
\hline Ashley & Levu & 8 & 10 & 0.607 & 46 \\
\hline Lauren & Yawa & 3 & 7 & 0.63 & 39 \\
\hline Joe & Soko & 12 & 6 & 0.607 & 26 \\
\hline JP & Levu & 6 & 8 & 0.586 & 25 \\
\hline Cole & Soko & 7 & 4 & 0.531 & 26 \\
\hline Desi & Soko & 11 & 3 & 0.515 & 9 \\
\hline Jessica & Soko & 7 & 1 & 0.415 & 6 \\
\hline Ali & Yawa & 3 & 4 & 0.5 & 19 \\
\hline Roark & Soko & 3 & 1 & 0.415 & 6 \\
\hline Alan & Levu & 2 & 2 & 0.415 & 11 \\
\hline Patrick & Yawa & 5 & 2 & 0.405 & 6 \\
\hline Simone & Yawa & 5 & 1 & 0.293 & 4 \\
\hline Katrina & Levu & 5 & 1 & 0.386 & 5 \\
\hline
\end{tabular}

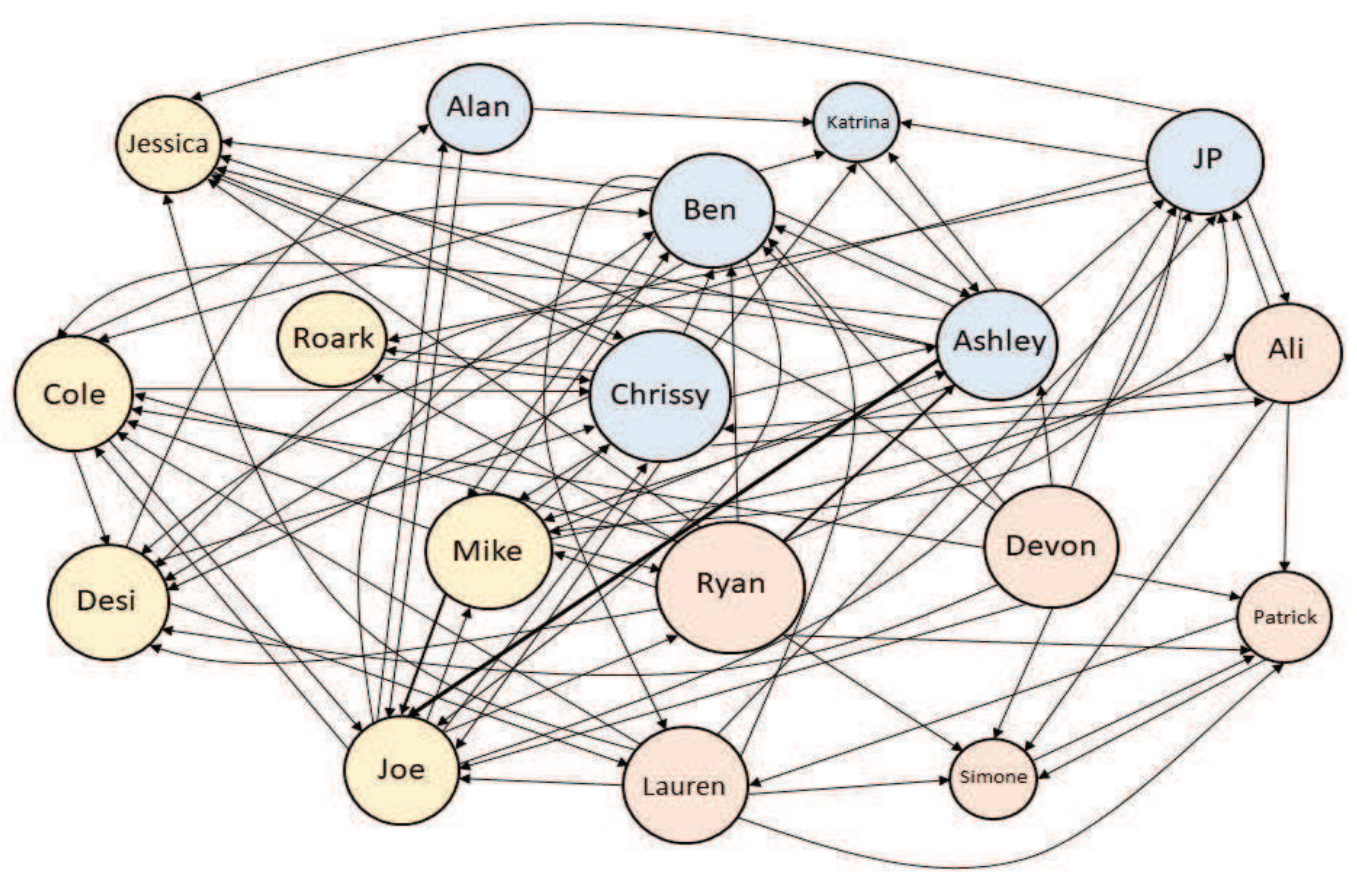

FiguRE 3.4. Survivor: HHH. 


\subsection{Big Brother}

We now move to data from Survivor to another social game show: Big Brother. We only focus on Big Brother 12 which is the twelfth season of the U.S. version of the reality show. There were thirteen HouseGuests in this season and they spent seventy five days. Hayden, Enzo, Lane, and Matt formed an alliance called The Brigade. Hayden and Lane secured their place in the final two. The jury voted to give Hayden the grand prize by a vote of 4 to 3 . Hayden received half a million dollars for spending seventy five days in the Big Brother House.

TABle 5. Big Brother, Season 12.

\begin{tabular}{|l|l|l|l|c|}
\hline Name & ID & OD & C & CON \\
\hline \hline Hayden & 3 & 16 & 0.923 & 44 \\
\hline Lane & 3 & 10 & 0.857 & 46 \\
\hline Enzo & 4 & 9 & 0.8 & 48 \\
\hline Britney & 4 & 10 & 0.8 & 43 \\
\hline Regan & 5 & 8 & 0.706 & 49 \\
\hline Brendon & 7 & 9 & 0.706 & 40 \\
\hline Matt & 9 & 7 & 0.632 & 35 \\
\hline Kathy & 7 & 4 & 0.6 & 20 \\
\hline Rachel & 8 & 6 & 0.667 & 24 \\
\hline Kristen & 7 & 3 & 1 & 25 \\
\hline Andrew & 9 & 2 & 1 & 17 \\
\hline Monet & 8 & 1 & 1 & 10 \\
\hline Annie & 11 & 0 & 0 & 0 \\
\hline
\end{tabular}

In Table 5, we note that Hayden, the winner of the season, had highest closeness and also the one of the high CON scores, with HouseGuests Lane and Enzo rounding out the top three. Lane and Enzo emerged also as leaders based on their scores. 


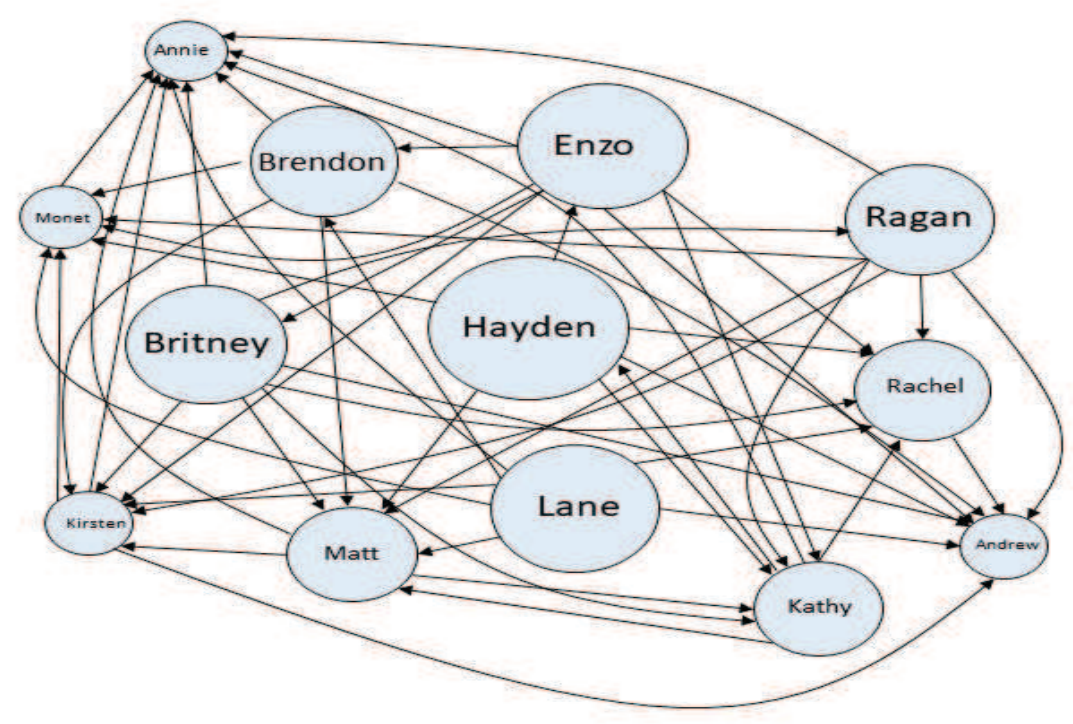

Figure 3.5. Big Brother, Season 12.

We discussed and analyzed the five seasons of two reality different shows, it is clear that the data conforms to the predictions of the Dynamic Competition Hypothesis (DCH) with respect to leaders which may be the winner of the game. We also found that the closeness is more predictive centrality measure than the betweenness in dynamic competition networks.

\subsection{Alliances}

In this section, we analyzed the alliances of four seasons from Survivor and one season from Big Brother. We computed their edge densities for predicting the winner in the game. We see that all alliances are near independent sets (with stronger alliances showing lower edge density), and they conform to the Dynamic Competition Hypothesis. 
TABLE 6. Edge densities of the Alliances.

\begin{tabular}{|l|l|l|l|l|}
\hline Season & Winner & Finalists & Alliances & ED \\
\hline Borneo & Richard & Kelly & $\begin{array}{l}\text { Barbecue: Colleen, Jenna, Ger- } \\
\text { vase } \\
\text { Tagi: Richard, Rudy, Susan, } \\
\text { Kelly }\end{array}$ & 1.667 \\
\hline China & Todd & Courtney & $\begin{array}{l}\text { Fei Long: Todd, Courtney, } \\
\text { Amanda, Aaron, Denise, James, } \\
\text { Frosti }\end{array}$ & 0.667 \\
Zhan Hu: Peih-Gee, Erik, Jaime & 0.0 \\
\hline $\begin{array}{l}\text { Game } \\
\text { Changers }\end{array}$ & Sarah & Brad & $\begin{array}{l}\text { Power Six: Sarah, Brad, } \\
\text { Troyzan, Sierra, Debbie, Tai } \\
\text { Tavua: Aubry, Cirie, Michaela, } \\
\text { Ozzy, Andrea, Zeke, Sarah }\end{array}$ & 0.933 \\
\hline Broyzan & Ben & Rhrissy & $\begin{array}{l}\text { Healers: Joe, Desi, Jessica, Cole, } \\
\text { Mike } \\
\text { The Round Table: Chrissy, Ryan, } \\
\text { Devon, JP, Ben, Ashley, Lauren } \\
\text { Final Four: Ashley, Lauren, Ben, }\end{array}$ & 0.905 \\
Devon \\
\hline BB 12 & Hayden & Lane & $\begin{array}{l}\text { The Brigade: Enzo, Hayden, } \\
\text { Lane, Matt }\end{array}$ & 0.5 \\
\hline
\end{tabular}

In Table 6, we provide the edge densities of each alliance in the aforementioned five seasons. We note that some alliances have high edge density as compared to other alliances. Although, when we remove the edges of those contestants who play against their alliance, then the alliance has lower edge density. For Example, in Survivor: Borneo, the edge density of the Tagi alliance is 1.5 but when we narrow down the alliances to subsets of finalist, then the edge density decreases: that is, the edge density of the subset $\{$ Kelly,Richard $\}$ is 1 and $\{$ Richard,Rudy $\}$ is 0. In the Fie-Long alliance in Survivor: China, the subset $\{$ Amanda, Courtney, Todd $\}$ has edge density 0 . We also see that in Round Table 
alliance in Survivor: HHH, the subset $\{$ Chrissy, Ryan, Devon $\}$ has edge density 0 . 


\section{CHAPTER 4}

\section{Conclusion and Future Work}

\subsection{Conclusion}

We introduced the notion of dynamic competition networks and studied their properties. In Chapter 2, we introduced structural balance theory. We defined a model of positive and negative relationships in a complete graph. We discussed, with examples, the balanced and unbalanced graphs in signed social networks. We also generalized the definition of signed complete graphs for groups of three nodes into an arbitrary numbers of nodes. We defined standard centrality metrics in network science and some graph theocratic tools that were used to state of Dynamic Competition Hypothesis (DCH). We formulated and presented the Dynamic Competition Hypothesis (DCH) which resolves dynamic competition networks arising from social networks into alliances, detects leaders, and measures the relative strength of alliances.

In Chapter 3, we discussed the background of U.S. reality television social game shows Survivor and Big Brother. We focused and analysed four seasons from Survivor and one season from Big Brother. We assessed the Dynamic Competition Hypothesis (DCH) with voting data for these seasons by using tools from network science. We observed that the DCH 
predicted the alliances corresponding to near independent sets, CON scores accurately determine leaders of alliances, and leaders were detected via their CON scores and closeness.

\subsection{Future Work}

In our thesis, we only analysed four seasons from U.S. Survivor and one season from Big Brother. In future work, we will extract data from all U.S. and international seasons of Survivor and Big Brother. We will also verify the DCH more extensively for different data sets of Survivor and Big Brother within the lens of structural balance theory and social network analysis. A weakness with the hypothesis is that contestants who remain longer stay in a season acquire more prestige simply because of their survival. In particular, we note that all those players in Survivor and Big Brother who remain long in the game, have a greater chance to increase their CON-scores and other metrics.

In future work, we will analyse data at earlier stages of the formation of the network. We can examine the DCH in the other fields; for examples, we will consider food webs, geo political networks and subgraphs (with negative ties) of the signed networks.

It would be intriguing to invert the $\mathrm{DCH}$ to determine the low ranked members of dynamic competition networks. Additionally, it would be convenient to develop a mathematical model anticipating the evolution of 
dynamic competition networks, which demonstrably simulate properties anticipate by the DCH. 


\section{Bibliography}

[1] M. Bastian, S. Heymann, M. Jacomy, Gephi: an open source software for exploring and manipulating networks, In: Proceedings of the International AAAI Conference on Weblogs and Social Media, 2009.

[2] A. Bevelas, Communication patterns in task-oriented groups, The Journal of the Acoustical Society of America 22(6): 725-730, 1950.

[3] Big Brother Wiki http://bigbrother.wikia.com/wiki/Big_Brother_Wiki.

[4] V. Boginski, S. Butenko, P.M. Pardalos, On structural properties of the market graph, In: A. Nagurney, editor, Innovation in Financial and Economic Networks, Edward Elgar Publishers, pp. $29-45$.

[5] A. Bonato, A Course on the Web Graph, American Mathematical Society Graduate Studies Series in Mathematics, Providence, Rhode Island, 2008.

[6] U. Brandes, T. Erlebach (Eds.) Network Analysis: Methodological Foundations, LNCS 3418, Springer, 2005.

[7] D. Cartwright, F. Harary, Structure balance: A generalization of Heider's theory, Psychological Review 63(5): 277-293, 1956.

[8] J.A. Davis, Structural balance, mechanical solidarity, and interpersonal relations, American Journal of Sociology 68 (1963) 444-462.

[9] D. Easley, J. Kleinberg, Networks, Crowds, and Markets Reasoning about a Highly Connected World, Cambridge University Press, 2010.

[10] L.C. Freeman, S.P. Borgatti, D.R. White, Centrality in valued graphs: A measure of betweenness based on network flow, Social Networks 13(2): 141-154, 1991.

[11] M.M. Hakeem, K. Suzuki, Centrality Measures of Trade and Investment Networks, Australian Academy of Accounting and Finance Reveiw 1(2): 103-118, 2015.

[12] F. Harary, On the notion of balance of a signed graph, Michigan Mathematical Journal 2(2): $143-146,1953$.

[13] F. Heider, The Psychology of Interpersonal Relations, John Wiley \& Sons, 1958. 
[14] F. Heider, Attitudes and cognitive organization, Journal of Psychology 21 (1946) 107-112.

[15] A.N. Langville, C.D. Meyer, Who's \#1? The Science of Rating and Ranking. Princeton University Press, 2012.

[16] Survivor Wiki http://survivor.wikia.com/wiki/Main_Page.

[17] S. Wasserman, , K. Faust, Social Network Analysis: Methods and Applications, Cambridge University Press, 1995.

[18] D.B. West, Introduction to Graph Theory, 2nd edition, Prentice Hall, 2001. 